\title{
Der psychophysische Parallelismus
}

\section{Zu einer Diskursfigur im Feld der wissenschaftlichen Umbrüche des ausgehenden 19. Jahrhunderts}

\author{
Mai Wegener
}

Psychophysical Parallelism. On a Discursive Figure in the Field of Scientific Changes in the late 19th Century

The article traces the rise and fall of "psychophysical parallelism" - which was the most advanced scientific formulation of the mind / body relationship in the second half of the 19th century - through an interdisciplinary and broad geographical spectrum. It sheds light on the extremely different positions that rallied round this discursive figure, ranging from Fechner, Hering, Mach, Wundt, Bain, Hughlings Jackson, and Taine to Freud and Saussure. The article develops the thesis that the psychophysical parallelism functioned as a 'hot zone' within and a symptom of the changes in the order of sciences at that time. Against that background, the criticism of the psychophysical parallelism which became prominent around 1900 (Stumpf, Busse, Bergson, Mauthner et. al.) indicates the cooling of this 'hot zone' and the establishment of a new order within the scientific disciplines. The article pays particular attention to the position of this figure in contemporaneous language theories. Its basic assumption is that the relationship between the body and the psyche is itself constituted by language.

Keywords: mind-body-problem, parallelism, psycho-physics, psychology, language

Schlüsselwörter: Leib-Seele-Problem, Parallelismus, Psychophysik, Psychologie, Sprache

Es ist ein Unterschied, ob man mit dem Gehirne denkt, oder in das Gehirn des Denkenden hineinsieht. Da erscheint ganz Verschiedenes. (Fechner 1860, Bd. 1: 4)

Unter dem Lemma Parallelismus, psychophysischer findet sich im Brockhaus von 1895 ein Eintrag, in dem dieser vorgestellt wird als „die Lehre, dass psychische Vorgänge regelmäßig von physischen begleitet werden“. „Danach erwächst dem Forscher die Aufgabe“, heißt es weiter, „zu einem gegebenen Bewusstseinsprozess den nervösen Parallelvorgang aufzusuchen." (Anonym 1895: 887) In dem kurzen Artikel wird der Parallelismus als heuristisches Prinzip präsentiert und die Auslegung favorisiert, dass ,jede der beiden Reihen, die physische wie die psychische, [...] dabei als eine in sich geschlossene, selbstständige aufgefasst“ (ebd.) wird. Die Formulierung war damals berühmt 
und verbreitet, sie galt als die wissenschaftlich avancierteste Auffassung des Verhältnisses von Psyche und Physis und genoss große Autorität. Dies gilt ungefähr für die Zeit zwischen 1850 und 1900, danach jedoch begann ihr Stern zu sinken. In verschiedenen Kontroversen wurde der Parallelismus um 1900 stark angegriffen und verlor seinen Stand als vorherrschende und breit diskutierte Figur.

Verschwunden ist der Parallelismus bis heute dennoch nicht. ${ }^{1}$ Forschungsansätze, die zu psychischen Prozessen den ,nervösen Parallelvorgang aufsuchen', haben derzeit eine gewaltige Konjunktur. Wesentlich befördert von den neuen bildgebenden Verfahren haben die Neurowissenschaften große Erfolge verbucht und zahlreiche Debatten ausgelöst. Allerdings ist der Parallelismus dabei kaum Gegenstand von Kontroversen geworden. Auch Titel wie Das parallelistische Fehlverständnis des Physischen und des Psychischen (Pohlenz 1977) oder Die Parallelität von Gehirn und Seele (Linke/Kurthen 1988), die immerhin anzeigen, dass die Figur nicht ganz verschwunden ist, konnten sie nicht wirklich in die Diskussion zurückbringen. Sie scheint eher implizit als eine allgemein bereit liegende Formulierung des Verhältnisses von Körper und Seele mitzulaufen. Der Fokus und das Vokabular der Debatten haben sich dabei in andere Richtungen verschoben. Die Figur des Parallelismus ist im heutigen Diskurs somit eher stumm gegenwärtig; als widerlegt gilt sie nicht.

Bei allen Unterschieden ist den Diskussionen damals und heute das Ringen um das Verhältnis von Natur- und Geisteswissenschaften gemeinsam, das mal mehr, mal weniger direkt mit der Diskussion um das Verhältnis von Psyche und Physis verknüpft ist. Allerdings ist die Gegenüberstellung von Natur- und Geisteswissenschaften erst im 19. Jahrhundert etabliert worden. Zuvor waren wissenschaftliche Praxis und Fächerordnung nicht auf diese Zweiteilung bezogen gewesen, und es ist keineswegs sicher, dass dies in Zukunft so bleiben wird. Dass auch heute diese Abgrenzungen weder stabil noch klar gezogen sind, davon zeugen die erneut vorgetragenen Gebietsmarkierungen - etwa das Manifest der Hirnforscher (Elger u.a. 2004) oder das der Geisteswissenschaftler (Gethmann u.a. 2005). Mit meiner historischen Untersuchung möchte ich dazu beitragen, die Aufmerksamkeit für die gegenwärtigen Problematisierungsweisen in diesem Feld zu schärfen. Nicht zuletzt bildet die damalige Diskussion um die Figur des psychophysischen Parallelismus inmitten der Etablierung der neuen Fächerordnung einen Teil des Grundgemäuers, auf dem die heutige Forschungslandschaft errichtet wurde.

\section{Der psychophysische Parallelismus als eine ,heiße Zone'}

Das Bekenntnis zum psychophysischen Parallelismus zirkulierte im 19. Jahrhundert weit gestreut in den Wissenschaften, aber auch darüber hinaus. Er 
wurde ebenso in der Philosophie wie auch in ästhetischen Schriften diskutiert. Im engeren Sinne griff man die Formulierung aber vor allem in den sich neu konstituierenden Fächern Neurologie, Sinnesphysiologie und Experimentalpsychologie auf. Der Londoner Neurologe John Hughlings Jackson hielt 1884 die allgemeine Akzeptanz des psychophysischen Parallelismus fest (für die sich im Englischen auch die Bezeichnung doctrine of concomitance verbreitet hatte) und nannte dabei Philosophen, Physiker, Biologen und Mediziner als seine Gewährsmänner:

It seems to me that the doctrine of concomitance is, at any rate, convenient in the study of nervous diseases. It, or essentially similar doctrine, is held by Hamilton, J.S. Mill, Clifford, Spencer, Max Müller, Bain, Huxley, Du Bois Reymond, Laycock, Tyndall, Hermann, and David Ferrier. (Jackson 1927 [1884]: 122)

In Deutschland war die Parallelismus-Figur besonders unter den Sinnesphysiologen weit verbreitet. Ewald Hering zählte neben Ernst Mach zu ihren nachdrücklichsten Verfechtern:

Unsere ganze Psychophysik fußt aber auf der Annahme, daß zwischen physischem und psychischem Geschehen ein gewisser Parallelismus bestehe, und daß insbesondere verschiedenen Qualitäten der Empfindungen auch verschiedene Qualitäten oder Formen des psychophysischen Geschehens entsprechen. (Hering 1878: 76)

Hering wollte dabei den Parallelismus als „conditio sine qua non aller solchen Forschung" (ebd.) verstanden wissen. Andere dagegen betonten, dass sie den psychophysischen Parallelismus als eine „didaktisch bequeme und heuristisch förderliche Formulierung eines Problems, nicht als eine Problemlösung" (Ziehen 1923: 226) ansehen. Die Formulierung stammt in diesem Fall von Theodor Ziehen, dessen Leitfaden der physiologischen Psychologie sich gleich eingangs für die Seite der "hirnphysiologische[n] Parallelvorgänge“ (Ziehen 1911 [1890]: 2), und allein für diese, zuständig erklärte. So war der psychophysische Parallelismus auch in die psychopathologische Praxis eingezogen und wurde zum Bezugspunkt für Psychiater, Aphasieforscher und Psychologen. Auf Seiten der Psychologen beteiligte sich Wilhelm Wundt besonders nachdrücklich an der Debatte. Er setzte 1894 die Formel des psychophysischen Parallelismus in den Titel einer 100-seitigen Abhandlung, in der er seine Auffassung des Parallelismus gegen die der Physiologen verteidigte, also interne Differenzen ausarbeitete (auf die später noch genauer eingegangen wird).

Die Berufung auf den Parallelismus verblieb aber nicht im Rahmen der Naturwissenschaften beziehungsweise der sich wissenschaftlich neu orientierenden Medizin und Psychologie. Die Debatte erfasste weite Kreise der Kultur, denn sie war Ausdruck und Teil einer grundlegenden wissenschaftlichen Umorientierung: der Physikalisierung des Lebens - die auch das psychische Leben ergriff. Das ,Zeitalter der Nerven' hat man die zweite Hälfte des 19. Jahrhunderts treffend genannt, und dies nicht allein aufgrund der damals so intensiv beforschten ,Volkskrankheiten' der Nervosität, Neuras- 
thenie und Hysterie (Radkau 1998, Steiner 1964), sondern weil auf einer allgemeinen Ebene die Nerven zu einem Einsatz im Wahrheitsspiel ${ }^{2}$ wurden. Dabei war der Bezug auf die Neurophysiologie in Fragen des Psychischen als solcher nicht neu. Aber er war, durch die zunehmend experimentelle und apparative Beforschung der Nerven und insbesondere des Gehirns, von einer neuen Qualität und Nachdrücklichkeit. Der erfolgreiche Nachweis der tierischen Elektrizität (Emil Du Bois Reymond) und der Elektrizität des Gehirns (Eduard Hitzig/Gustav Theodor Fritsch), die ersten Messungen der Nervenleitgeschwindigkeit (Hermann von Helmholtz) und der Ausbau der Reflexforschung taten ihr Übriges, um die Präsenz des ,Nervenapparates im allgemeinen Diskurs zu erhöhen. Davon blieben auch Kunst und Literatur nicht unberührt (Worbs 1983). Auch wenn der explizite Bezug auf die Formel des psychophysischen Parallelismus in der Kunst eine Sache von wenigen war, war sie dort besonders attraktiv, wo der Wissenschaftsbezug emphatisch vorgetragen oder wo eine wissenschaftliche Fundierung der Ästhetik angestrebt wurde. So wurde in der Russischen Akademie der Kunstwissenschaften in Moskau in dem dort maßgeblich von Kandinsky begründeten psychophysischen Labor intensiv Georgy Chelpanovs „empirische[r] Parallelismus" rezipiert, den dieser in Anlehnung an Wundt proklamierte (Misler 1997: 27). Einen durchaus amüsanten Zug hatte Wilhelm Bölsches Aufnahme der Figur in seine Naturwissenschaftlichen Grundlagen der Poesie von 1886. Die ausdrückliche Berufung auf den „Parallelismus des Psychischen und des Molekularen" (Bölsche 1976 [1886]: 37, vgl. 53f.) diente ihm als Stütze der Forderung, die körperliche Liebe möge von den Literaten ebenso berücksichtigt werden wie die seelische. Bölsche mahnte die Schriftsteller, auch das körperliche Liebesleben von der Jugend bis ins Alter darzustellen und nicht nur die göttliche und nervöse Liebe, die sie so gern besingen würden. Er führte dies 1889 in seinem Liebesleben in der Natur quer durch das Tierreich selbst vor.

Der durchgehende Zuspruch, den die Figur des psychophysischen Parallelismus erfuhr, ist beeindruckend. Dabei waren die Positionen, die sich unter ihrem Label versammelten, jedoch keineswegs einheitlich, sondern höchst different. Und es war gerade diese Offenheit, die die umfassende Zustimmung beförderte: Die Figur eröffnete einen Raum, in dem sich die Positionen treffen konnten, bevor von verschiedenen Seiten Präzisierungen vorgenommen wurden, die dann nicht mehr miteinander vereinbar waren.

Zunächst und grundlegend war der psychophysische Parallelismus eine Figur der Absetzung. Mit ihr wurden die historischen Vorgängermodelle zur Beschreibung des Verhältnisses von Körper und Seele verworfen (Welsh 2003: 19f.). In diesem Punkt herrschte die größte und am klarsten vorgebrachte Einigkeit. Die Formulierung des Parallelismus stellte sich gegen die bereits im Fall befindliche Konzeption des ,Seelenorgans', nach der die Seele an einem bestimmten, wie auch immer gearteten Punkt mit dem Hirn 
verknüpft sei. Dies war die dem Descart'schen Paradigma zugehörige Vorstellung, deren Zerfall Michael Hagner (1997) und Olaf Breidbach (1997) in ihren Arbeiten nachgezeichnet und als Vorbedingung für die Entfaltung der modernen Hirnforschung gekennzeichnet haben. Mit der Verabschiedung des Descart'schen Dualismus wurde die Vorstellung einer ausgedehnten Seele denkbar und damit ein materiell fundierter ,Seelenapparat'. Spezifischer noch wurde dabei die Formulierung des psychophysischen Parallelismus der als obsolet geltenden Vorstellung einer Wechselwirkung zwischen Leib und Seele entgegengesetzt. Letztere war mit dem Konzept des ,Seelenorgans' gut vereinbar gewesen; nach ihr wirkt das Seelische auf das Physische - oder umgekehrt. Entscheidend ist, dass beides in einem Verhältnis von Kausalität zueinander gedacht wurde. Genau dies aber wurde jetzt unter Hinweis auf das Gesetz von der Geschlossenheit der Naturkausalität zurückgewiesen, das mit der Physik als Leitwissenschaft auch in den Lebenswissenschaften zum unumgänglichen Grundsatzes geworden war: Allein Materie wirke auf Materie. Etwas Nicht-Materielles wie das Bewusstsein oder das Psychische könne demnach Materie nicht beeinflussen. ${ }^{3}$ Körperliche Vorgänge, so betonte man daher, hören nicht in dem Moment auf, sobald Bewusstseinsphänomene auftreten, beide laufen gleichzeitig - eben parallel - zueinander und stehen in keinem kausalen Verhältnis.

Die Kennzeichnung der Arbeiten der romantischen Naturforscher als unwissenschaftlich und unpräzise stammt aus dieser Mitte des 19. Jahrhunderts einsetzenden Abgrenzungsbewegung. An die Stelle der Naturphilosophie sollte Naturwissenschaft treten. In diesem Zuge wurde die rege experimentelle Praxis der anthropologischen Naturforscher und Mediziner, etwa die Experimente Wilhelm Ritters und Johann Christian Reils, abgewertet oder übergangen (Welsh 2003). Allerdings stellte die Formulierung des Gesetzes von der Erhaltung der Kraft durch Joule, Mayer und schließlich durch Helmholtz in physikalisch strenger Form einen tatsächlichen Bruch dar. Sie gab den physiologischen Erörterungen eine neue Grundlage, die mit der Hoffnung auf eine mit der Physik vergleichbare Strenge verbunden war (vgl. Lenoir 1997: 75f.). Die Formulierung des psychophysischen Parallelismus hatte in dieser Umbruchszeit ihren Platz auf der Seite der Trennung von der Naturphilosophie und stand für eine wissenschaftliche Neubegründung der Fächer.

Die Differenzen begannen allerdings, sobald der positive Gehalt der Figur näher bestimmt werden sollte. Inhaltlich bildeten sich deutlich verschiedene Auslegungen des Parallelenbildes heraus. Je nachdem, ob die Kongruenz betont wurde oder die Tatsache, dass sich Parallelen nicht schneiden, stand die strenge Abhängigkeit der Sphären im Vordergrund oder ihre Differenz. Es gab Hierarchisierungen und verschiedene Bezugnahmen auf die Räumlichkeit der Metapher, auch wurde die Kausalität, deren Wechselwirkung aus- 
geschlossen war, nun innerhalb der psychischen oder physischen Reihe sehr unterschiedlich gefasst.

Bereits der Status der Figur in den zeitgenössischen Texten war höchst divers. Mal wurde der Parallelismus vorsichtiger als „Hypothese“ (Hippolyte Taine, Wilhelm Dilthey) eingeführt, mal bestimmter als "Lehre“ (John Hughlings Jackson) oder "Theorie“ (Alexander Herzberg). Gustav Theodor Fechner nannte ihn einen "Standpunkt“, auf den Ewald Hering dann die Forschung grundsätzlich verpflichten wollte. Besonders häufig wurde die Figur als "heuristisches Prinzip“ (Ernst Mach) oder „empirische Hypothese“ (Wilhelm Wundt) vorgestellt, was die allgemeine Hoffnung zum Ausdruck brachte, auf dem Wege der empirischen Forschung zu seiner Bestätigung gelangen zu können. Als Henri Bergson den Parallelismus 1904 als eine „idée“ bezeichnete, holte er die Figur in die Sphäre der Metaphysik zurück und brachte damit seinen polemischen Einsatz auf den Punkt, die Möglichkeit einer rein empirischen Lösung zurückzuweisen.

Diese Unterschiede in der Benennung sind bereits Ausdruck der spezifischen Auslegung der Figur. Dies gilt ebenso für die Platzierung der Überlegungen zum psychophysischen Parallelismus im Text: Mal wurde die Figur direkt in der Einleitung, gleichsam als Auftakt genannt, mal im Nachwort diskutiert - gelegentlich mit dem Zusatz, dass man jetzt den wissenschaftlichen Teil verlasse und persönliche Ansichten äußere -, oder sie wurde, wie bei Fechner, ausführlich im Haupttext selbst entwickelt. Erst in dem Moment, in dem ein offener Streit um die Figur entbrannte, wurde sie Gegenstand eigenständiger Abhandlungen.

In diesem Beitrag wird zumeist von der Figur des psychophysischen Parallelismus die Rede sein. Um eine Figur handelt es sich zunächst im geometrischen Sinne, insofern hier das geometrische Bild zweier zueinander parallel verlaufender Linien aufgenommen wird, um aus ihm eine gewisse, vielleicht auch nur vermeintliche Anschaulichkeit zu beziehen. In der Übertragung ist der Parallelismus aber vor allem eine sprachliche Figur. Er war die meistgebrauchte Metapher ${ }^{4}$ für den Zusammenhang von Körper und Psyche in den Texten des späten 19. Jahrhunderts. In diesem Beitrag liegt das Interesse allerdings weniger auf der Metaphorizität oder Bildhaftigkeit der Figur (Wegener 2008: 137f.), als auf ihrem Einsatz als Diskursfigur. Dazu wird eine Figur (die eine Metapher sein kann, aber nicht muss), wenn ihr innerhalb eines Diskurses eine bestimmte, diesen Diskurs markierende Funktion zukommt. Ihre Bedeutung lässt sich erst aus dem spezifischen Kontext heraus bestimmen, in dem sie gebraucht wird. Im Folgenden wird daher nach der Funktion gefragt, die die Figur des psychosphyischen Parallelismus in den verschiedenen Diskursen hatte, und nach den Strategien und Motiven, die sich an ihren Gebrauch jeweils knüpften. Im Zentrum der Aufmerksamkeit steht dabei ihr Einsatz in der Umordnung des Wissens. 
Wie man in Kontrastierung zu Bruno Latours Rede von der „erkalteten Wissenschaft" (Latour 1997) sagen könnte, bildete der psychophysische Parallelismus damals eine ,heiße Zone' (Wegener 2006: 65f., 2008: 131f.). Das heißt, er befand sich nicht auf der Seite der geronnenen wissenschaftlichen Ergebnisse, sondern markierte ein im Umbau begriffenes, umstrittenes Feld hoher Aktivität, eine ,heiße Zone', in der sich Fragen bündelten und Reibungshitze entstand. Der psychophysische Parallelismus stellte, so die These, ein Symptom der Auseinandersetzung zwischen den Disziplinen dar, wie man von einem Symptom als einer Kompromissbildung sprechen kann: Er stand für ein Problem, das er im selben Zug deckte - eine wirkliche Frage, die wie eine Antwort daherkam. Zugleich war der Parallelismus ein Symptom im Sinne eines Zeichens: Sein Auftauchen verwies auf einen umfassenderen Prozess. Die Figur gelangte zu einer solchen Blüte, weil sie die verschiedenen Parteien im Disziplinenstreit versammelte und sich dabei zugleich von ihnen als Kampfbegriff und Ausweis der neuen Wissenschaftlichkeit einsetzen ließ. Die Emphase erlosch in dem Moment, als die Physikalisierung des Lebens keine Frage mehr war, gleichsam erkaltete und die Umordnung des Wissens in den beteiligten Fächern - insbesondere die Etablierung der Neurologie und der Psychologie - vollzogen war. Erst dann brach interessanterweise die Debatte als philosophischer Streit aus. Seither bildet die Figur ein Hybrid aus wissenschaftlichem, philosophischem und Alltagsdiskurs.

\section{Parallelismus strictus existit}

Bevor sie im 19. Jahrhundert so prominent wurde, war die Figur des psychophysischen Parallelismus bereits von philosophischer Seite formuliert worden, so von Spinoza und Leibniz. In der zeitgenössischen Debatte grenzte man sich von beiden ab und ließ die Figur als selbstbewusste Neuformulierung auftreten: Es sollte nicht mehr um Philosophie gehen, sondern um eine empirisch gewonnene Formel. Bemerkenswerterweise wurde die Figur nicht von Naturwissenschaftlern eingeführt - für deren strenge Perspektive sie später einstehen sollte -, sondern von Denkern, die zwischen den sich damals erst herausbildenden Disziplinen standen. In Deutschland war es der Psychophysiker Theodor Fechner, der sie in verschiedenen Schriften zwischen 1823 und 1860 vorstellte und begründete. In England wurde sie 1865 und dann ausführlich 1872 von dem Aberdeener Logikprofessor und Mill-Schüler Alexander Bain eingeführt und in Frankreich 1870 von dem ansonsten als Professor für Kunstgeschichte und homme de lettres bekannten Promotor des Szientismus Hippolyte Taine. In Stil, Kontext und Stoßrichtung unterscheiden sich diese drei Auftakte, zwischen denen es gleichwohl Austausch und Überschneidungen gab. 


\section{Die Geburt des psychophysischen Parallelismus aus der Naturphilosophie}

Fechner war der erste im 19. Jahrhundert, der die Figur des psychophysischen Parallelismus ins Spiel brachte, und er behandelte sie am ausführlichsten. Sobald sie sich von Fechners Schriften löste, wandelte sich ihr Sinn allerdings schnell und radikal. Doch ihre erstaunliche Tragkraft verdankt sich in Deutschland zumindest zu einem Teil dem Kredit, den Fechner ihr gab, und wohl auch dem Versprechen von Einheitlichkeit der Welt, das er mit ihr transportierte. Bevor die Formulierung des Parallelismus mit den Elementen der Psychophysik 1860 bekannt wurde, hatte sie bereits einen festen Stand in seinen Werken. Erstmals findet sie sich 1823 in der Habilitation des damals 22-jährigen, noch von Schelling und Oken beeinflussten Studenten über die Prämissen einer allgemeinen Theorie der Organismen ${ }^{5}$ : „Parallelismus strictus existit inter animam et corpus, ita ut ex uno, rite cognito, alterum construi possit." (Fechner 1823: 23 [Es existiert ein strenger Parallelismus zwischen Seele und Körper, so dass aus dem einen, soweit erkannt, das andere konstruiert werden kann/Übers. M. W.]) Das Gewicht, das Fechner der Formel beimisst, lässt sich anhand der Stellung beurteilen, die er ihr in seinen beiden großen Werken gab. Nicht nur in den Elementen der Psychophysik, sondern bereits im Zend-Avesta kommt dem "faktischen Parallelismus des Körperlichen und Geistigen“ (Fechner 1851, Bd. 2: 365, vgl. 330, 347) eine tragende Funktion zu. Anders als die Elemente der Psychophysik - das weitaus bekanntere der beiden Bücher, ganz im Geist des neuen Szientismus verfasst - handelt der Zend-Avesta über die Dinge des Himmels und des Jenseits. Fechner dehnte hier seine Allbeseeltheitslehre von den Pflanzen (Fechner 1848) weiter auf die Planeten aus. Für ihn war Beseeltheit nicht an die Bedingung des organischen Lebens gebunden, sondern nur im allgemeinsten Sinne an das Vorhandensein von Körpern. Man sieht hier bereits die ebenso einfache wie erstaunliche Konsequenz des Parallelismus: Nicht nur alles Seelische hat eine materielle Seite, sondern auch alles Materielle eine seelische Seite.

Für den Autor gehörten beide Werke auf das engste zusammen (Wegener 2005), und es ist eben die Figur des psychophysischen Parallelismus, die die Brücke zwischen der naturwissenschaftlichen Vorder- und der esoterischen Rückseite von Fechners Arbeiten schlägt. Im Zend-Avesta heißt es eingangs: „Im Hintergrunde der ganzen Schrift liegt eine Grundansicht über die Beziehung von Leib und Seele oder von Körper und Geist." (Fechner 1851, Bd. 1: XVI) Fechner variiert in seinen Ausführungen die altehrwürdige Metapher vom Buch der Natur (Blumenberg 1998: 102-110, Kay 2000) und fasst Physis und Psyche als Schrift und Sinn:

Um mit einem Bilde zu beginnen, so ist das Leibliche oder Körperliche gleich einer Schrift, das Geistige, Psychische [...] wie der zugehörige Sinn der Schrift, in solcher Weise aber, daß die, als lebendig zu fassende Schrift sich selbst nur unter der Form ihres Sinnes, Andern 
nur unter der Form der äußeren Zeichen erscheinen kann, und daß beides nicht zufällig bei einander ist, wie in unseren Schriften, sondern in nothwendiger wesentlicher Beziehung zu einander. (Fechner 1851, Bd. 2: 313)

Entscheidend ist, dass der Schrift des Naturbuchs ein ihr "naturnothwendig“ zugehöriger Sinn beigegeben ist (ebd.). Hier liegt Fechners Akzent, denn dies entspricht seiner panpsychistischen Grundidee: Alle „Naturschrift“ hat Sinn, jeder Körper eine Seele (ebd.: 315). Der Parallelismus garantiert so die Beseeltheit und damit auch die Sinnhaftigkeit der Welt.

Fechners Grundauffassung ist die eines substantiellen Monismus. Naturschrift und Seelensinn sind für ihn zwei Seiten einer Medaille, Psyche und Physis sind „ein und dieselbe Sache“ einmal von innen, einmal von außen betrachtet (ebd.: 314, Heidelberger 1993: 135). Der Dualismus der Erscheinungen ergibt sich allein aus der Unmöglichkeit, beide Seiten oder Ansichten dieser Wesenheit auf einmal zu erfassen; er ist gleichwohl ebenso grundlegend wie der Monismus. Diese Unmöglichkeit bildet den Ausgangspunkt für das Defilee der Perspektivwechsel in Fechners Texten. Sein verdeutlichendes topologisches Bild kehrte in der Debatte oft wieder und wurde später auch heftig kritisiert (Stumpf 1897: 8, Mauthner 1923a [1901]: 287f., Busse 1903: 130f.): Wenn man im Inneren eines Kreises steht, erscheint sein Rand konkav, steht man dagegen auf der Außenseite desselben Kreises, ist der Rand nur von seiner konvexen Seite her zu sehen. So könne man irrtümlich dazu gelangen, die Seele (konkave Ansicht) für wesenhaft verschieden vom Körper (konvexe Ansicht) zu halten (Fechner 1851, Bd. 2: 343). Während Fechner dieses Bild zehn Jahre später in die Elemente der Psychophysik übernahm, war die Schriftmetapher aus seiner Darstellung verschwunden. Dabei hatte sie nicht nur esoterische Züge, Fechner setzte mit ihr auch, ganz modern, auf Kombinatorik:

Es kommt aber zum Ausdruck des Sinnes viel mehr auf die Zusammenstellung oder Folge der Zeichen und ihrer einfachen Kombinationen, Worte, als auf die Beschaffenheit der elementaren Zeichen und Worte selbst an, so daß mit denselben Elementen je nach ihrer Zusammenstellung ein sehr verschiedener Sinn ausgedrückt werden kann. D.h. dieselben körperlichen Elemente können je nach ihrer Zusammenstellung und Bewegung ein Geistiges von sehr verschiedener Art tragen. (Ebd.: 313f.).

Damit enthierarchisierte er die geistigen Funktionen. Durch die Mannigfaltigkeit der Kombinationen kann "höheres“ und „niederes“ Geistiges von derselben Schrift getragen werden, ganz wie in der Alphabetschrift das Werk eines Dichters ebenso wie irgendein Einkaufszettel notiert werden kann. Fechner fand mit seiner Parallelisierung von Schrift und Sinn außerdem ein Modell für die bindende, vereinheitlichende Kraft der Seele. Indem er jetzt mehrere Schriften (genauer Zahlenreihen) übereinander schichtete, veranschaulichte er seine Vorstellung vom „Stufenbau der Welt", nach der jede körperliche Stufe in einer höheren seelischen inbegriffen ist. Noch Gott ließ sich so für Fechner als innere Konsequenz des alles beseelenden Parallelismus darstellen: 
Erzeugt todte Schrift in Jemand einen Gedanken, kann sie es doch nur, sofern sie erst von einer gedankenvollen Bewegung ausging und noch einem höhern gedankenvollen Zusammenhange, in dem wir Alle mit der Schrift zugleich begriffen sind, angehört, und in ein gedankentragendes Gehirn hineinwirkt. (Ebd.: 318)

In Elemente der Psychophysik präsentiert Fechner dagegen vor allem Experimental- und Messergebnisse. Obwohl das Gewicht hier auf den „funktionellen oder Abhängigkeitsbeziehungen" zwischen Körper und Seele liegt (Fechner 1860, Bd. 1: 8), ist die Entfaltung des psychophysischen Parallelismus in den Hintergrund gerückt, denn er ist schon da. Seine Vorstellung nimmt nur noch acht Seiten ein, Fechner betont jetzt die Subjektivität seiner Ansicht, die er „ohne irgendwelche bindende Konsequenz" vorträgt: „Suche jeder sich das Rätsel, insofern es ihm als solches erscheint, auf seine Weise zu lösen." (ebd.: 3) Er vollzieht eine deutliche Trennung zwischen dem empirisch Zugänglichen und der Spekulation und verlangt wissenschaftliche Anerkennung nur für das auf „rein empirischer Grundlage“ Gewonnene (ebd.: 7). Im Vordergrund steht nun das Verhältnis von Reiz und Empfindung, dem Fechner eine mathematische Formel gab, die er über Jahre hinweg mit Weber gemeinsam erarbeitet hatte (Heidelberger 1993: 220-237). Danach vermittelt zwischen Reiz und Empfindung ein innerer körperlicher Prozess, der die vom physikalischen Reiz ausgelöste psychische Empfindung trägt. Fechner nannte ihn den „psychophysischen“ und räumte ein, dass dieser Prozess in seiner ganzen Beschaffenheit eine Unbekannte bleibe. Im Zentrum der Empirie der sogenannten „äußeren Psychophysik“ lag somit ein Bereich, über den nur zu spekulieren war. Die hiermit befasste „innere Psychophysik“ lag Fechner zwar am Herzen, beanspruchte aber nicht denselben wissenschaftlichen Rang wie das im Rahmen der „äußeren Psychophysik“ aufgestellte Weber-Fechner'sche Grundgesetz über das logarithmische Verhältnis von Reiz und Empfindung.

Fechner reflektierte die Stellung, die seine Arbeiten im epistemischen Feld einnahmen, in beiden Hauptschriften aufmerksam. Er hielt dabei fest, dass sich seine Untersuchungen weder eindeutig auf der Seite der Naturwissenschaften einordnen lassen - da diese allein die physische, äußere Welt im Blick hätten - noch auf der Seite der Wissenschaften vom Geiste, die sich nur auf den inneren Standpunkt der Betrachtung beschränken würden. Für seine Arbeiten nahm er einen dritten, von ihm ebenfalls als wissenschaftlich bezeichneten Zugang in Anspruch, den er den "naturphilosophischen“ nannte. Dessen Perspektive sei eine verknüpfende, die „beide Standpunkte combinierend, die materielle und die ideelle Seite in consequenter Beziehung auf einander verfolgt" (Fechner 1851, Bd. 2: 361, vgl. 1860, Bd. 1: 6). ${ }^{6}$ An seiner Stellung zur Naturphilosophie wird deutlich, dass Fechner die Absetzungsprobleme, die nach ihm so beherrschend wurden, noch nicht hatte. Als Begründer der Psychophysik war er selbst kein Materialist, sondern Befürworter eines „objektiven Idealismus“ (Fechner 1879: 240). Er stützte 
dabei seine nicht-materialistische Position bemerkenswerterweise auf den Parallelismus - also auf die Figur, die später vielfach als Marke der naturwissenschaftlich-materialistischen Position gelten sollte. Nachdem Fechner erklärte, Bewegung und Materie „stellen den Buchstaben, das Wort des Gedankens, der Empfindung vor“, stellte er ausdrücklich klar: „Diese Vorstellungsweise mag für den ersten Augenblick materialistisch erscheinen; ist es aber nicht." (Fechner 1851, Bd. 2: 317f.) Der Parallelismus kappt die Vorstellung einer Kausalität, die die Gedanken zum Produkt materieller Bewegung machen würde. Letztere trägt, aber sie erzeugt die Gedanken nicht: „Nur eine gedankenvolle Bewegung vermag wieder eine gedankenvolle Bewegung zu erzeugen; also fließt nach uns nicht Geist aus Materie." (Ebd., vgl. ebd.: 344)

Die Rezeption von Fechners Parallelismus schloss an die Elemente der Psychophysik an, nicht an den Zend-Avesta. Dadurch wurde der psychophysische Parallelismus weitaus stärker mit der experimentell-wissenschaftlichen Herangehensweise verknüpft als dies bei Fechner der Fall war. Tatsächlich wurde der Parallelismus erst in der Rezeption zu einem Einsatz im Wissenschaftsstreit, der Fechner selbst noch gänzlich fern lag. Zu den ersten Rezipienten gehörte der Physiker Ernst Mach, der 1863 in seinen Vorträgen über Psychophysik den sich schnell ausbreitenden Eindruck formulierte, dass Fechners Aufstellung eines „gesetzmäßigen Zusammenhangs zwischen Reiz und Empfindung“ ein "genialer Gedanke“ sei (Mach 1863: 226). Das WeberFechner'sche-Grundgesetz wurde allgemein diskutiert und geprüft. Auch wenn es bisweilen scharf angegriffen wurde, traf es einen Nerv und galt als Grundidee. Der Physiologe und Fechnerschüler Ewald Hering machte sich 1875 daran, die Unhaltbarkeit von Fechners logarithmischem Gesetz im Einzelnen nachzuweisen, zielte mit seiner Attacke aber keineswegs auf eine Abkehr von der grundsätzlichen Hypothese des gesetzmäßigen Zusammenhanges, sondern rang um dessen richtige Fassung (Hering 1875). Er war, wie bereits erwähnt, ein strenger Verfechter der Formel des psychophysischen Parallelismus. Hering hielt Fechner entgegen, „zu wenig Anwendung von derselben“ zu machen und setzte fort:

\footnotetext{
Mach bezeichnet diese Grundvoraussetzung der ganzen Psychophysik als blos „ein heuristisches Prinzip der psychophysischen Forschung“, aber sie ist mehr, sie ist die conditio sine qua non aller solchen Forschung, wenn sie Früchte tragen soll. (Hering 1878: 76)
}

Tatsächlich hatte Mach 1863 den psychophysischen Parallelismus als heuristisches Prinzip vorgestellt. 1886 bezeichnete er ihn dann in seiner Analyse der Empfindungen, die "das Verhältnis des Physischen zum Psychischen“ ausdrücklich zum Gegenstand hatte, als „leitenden Grundsatz“ und „notwendige Voraussetzung der exakten Forschung" (Mach 1906 [1886]: 50). Von Fechners metaphysischem Untergrund distanzierte sich Mach als Positivist, übernahm aber den Monismus beziehungsweise Identitätsgedanken 
und den Ansichtsdualismus (Heidelberger 2000: 61): „Ich sehe daher keinen Gegensatz von Psychischem und Physischem, sondern einfach Identität in Bezug auf diese Elemente“, heißt es bei Mach (1906 [1886]: 36). Als „Elemente" bestimmte er die Empfindungen in ihrer doppelten Eigenschaft als psychische und physiologische Phänomene. Um seine neue Perspektive einzuführen, wich Mach jetzt von dem bislang befolgten Grundsatz ab, den Parallelismus erst am Ende einer Erörterung zu diskutieren (gleichsam als erhellende, aber nicht notwendige Hypothese), sondern stellte „das Prinzip des vollständigen Parallelismus des Psychischen und Physischen" gleich in den Anfangskapiteln vor:

Nach unserer Grundanschauung, welche eine Kluft zwischen den beiden Gebieten (des Psychischen und des Physischen) gar nicht anerkennt, ist dieses Prinzip fast selbstverständlich. (Ebd.: 50)

Mach stützte seinen Einsatz, nach dem die Empfindungen, genauer, „die Farben, Töne, Räume, Zeiten ... [...] für uns vorläufig die letzten Elemente [sind], deren gegebenen Zusammenhang wir zu erforschen haben" (ebd.: 24), auf dieses Prinzip. Und von ihm aus führte er auch seinen Angriff gegen Kants „Ding an sich“ und die Substanzbegriffe der Metaphysik, wobei er besonders das „Ich“ im Visier hatte, dessen „Unrettbarkeit“ (ebd.: 20) er proklamierte. Mach und Hering leiteten die "physiologische Deutung der Psychophysik" (Scheerer 1993: 275) ein, die den Parallelismus linearer und materialistischer fasste als Fechner dies getan hatte. Dessen Anstrengung, mittels des Parallelismus Wissenschaft und Religion zu versöhnen, war nicht ihre Sache.

\section{Close Incorporation of Two Extreme and Contrast Facts}

Um die Unterschiedlichkeit der Interpretationen zu erfassen, die die Figur zuließ, muss man sich ihrer Gebrauchsweise in Großbritannien und Frankreich zuwenden. Der Neubegründer der Parallelismuslehre in Großbritannien war Alexander Bain. Wie Fechner war er einer der Universalgebildeten seiner Zeit: Professor für Logik und englische Literatur in Aberdeen, nachdem ihm eine Professur für Mathematik und natural philosophy in Glasgow als zu eng erschien. Bereits sein erstes umfangreiches Buch The Senses and the Intellect, das 1855 im selben Jahr mit Herbert Spencers Principles of Psychology erschien, kündigte Bains Interesse an einer von Nerven- und Hirnphysiologie erneuerten Psychologie an, das als roter Faden sein Werk durchläuft und ihn 1876 zum Begründer der Zeitschrift Mind werden ließ. Bain wurde neben Spencer damit zu einem der wichtigsten Promotoren der modernen Psychologie in Großbritannien. Beide bekannten sich zum Parallelismus. Vor allem Bain entwickelte eine Version des psychophysischen Parallelismus in Mind and Body (1872). Er formulierte die Figur in einem Umfeld, in dem bereits intensiv an der Verbindung von physischen und psy- 
chischen Vorgängen geforscht wurde. So konnte er sich auf die Arbeiten der britischen Ärzte und Neurologen ebenso beziehen wie auf die französischen und deutschen Forscher, welche er persönlich von seinen Reisen kannte. Auf Fechner bezog er sich nicht.

Den entscheidendsten Einfluss auf Bains Stellung zu dem Problem hatte John Stuart Mill, mit dem Bain freundschaftlich und philosophisch verbunden war. Mill hatte 1843 im letzten Buch seiner Logik, das den moral sciences (Geisteswissenschaften) gewidmet war, die Frage aufgeworfen, ob die Gesetze des Geistes eigenständig oder von denen der Physiologie, also des Körpers, ableitbar seien. Davon hänge ab, ob es eine eigenständige Wissenschaft des Geistes geben könne. Mill ging von der Existenz solcher eigenständiger Gesetze aus und grenzte sich ausdrücklich von „viele[n] eminente[n] Physiologen“ (Mill 1877 [1843]: 466) ab, denen zufolge nie ein Geisteszustand durch einen anderen erzeugt würde und die also die Existenz ursprünglich geistiger Gesetze negierten. In ihrer Perspektive sei die Wissenschaft des Geistes „ein blosser Zweig [...] der Physiologie“ (ebd.: 467). Im Gegensatz dazu sprach Mill sich für eine Eigenständigkeit der moral sciences auf der Basis von Experiment und Beobachtung und im Austausch mit der Physiologie aus. In diesem Sinne führte Bain die Figur des psychophysischen Parallelismus mit dem Akzent auf der Differenz der beiden Naturen ein:

While admitting that there is something unique, if not remarkable, in the close incorporation of the two extreme and contrast facts, termed Mind and Matter, we must grant that the total difference of nature has rendered the union very puzzling to express in language. (Bain 1873 [1872]: 134)

Bain führte den Parallelismus also nicht als Monismus ein. Tatsächlich lobte er Descartes für seine treffliche Unterscheidung von Materie und Geist als ausgedehnte und unausgedehnte Substanz, auch wenn er die cartesische Theorie der Seele und des Seelensitzes zurückwies (ebd.: 192). Bain kam anders als Fechner vom Standpunkt des Dualismus her zur Formulierung des Parallelismus. Die Eigenschaften von Geist und Materie stehen, wie er betont, in schroffem Kontrast (ebd.: 124): „Our mental experience, our feelings and thoughts, have no extension, no place, no form or outline, no mechanical division in parts." (Ebd.: 135) Bain sprach sich daher auch gegen die Lokalisierung des Geistes aus. Es sei kein Ort angebbar, an dem er mit dem Körper verbunden sein sollte: „When, therefore, we talk of incorporating mind with brain, we must [...] yet deprive it of the almost invincible association of union in place." (Ebd.: 137) Gleichwohl kennen wir den Geist faktisch nur in Verbindung mit lebender oder organisierter Materie, hielt Bain fest, der nicht an beseelte Sterne glaubte (ebd.: 127). Er dachte die Verbundenheit aus diesem Grund nicht räumlich, sondern zeitlich: „The only mode of union that is not contradictory is the union of close succession in time; ore of position in a continued thread of conscious life." (Ebd.: 137) Wie allgemein üblich 
bestand auch für Bain der Parallelismus in der Zurückweisung jeglicher Kausalität zwischen Psyche und Physis. Eine solche Wechselwirkung liefe auf die Unterbrechung des physischen Wirkungskreislaufs hinaus, als gebe es „two shores of the material with an intervening ocean of the immaterial" (ebd.: 131). Er hielt dagegen die Kontinuität:

There is in fact no rupture of nervous continuity. The only tenable supposition is, that mental and physical proceed together, as undivided twins. When therefore we speak of a mental cause, a mental agency we have always a two-sided-cause. (Ebd.: 131)

Bain ging von zwei eigenständigen kausalen Vorgängen aus, die als eine doppelte „mind-body“-Ursächlichkeit in Erscheinung treten. Er nannte die Verbindung zwischen Psyche und Physis eine „correspondence, or concomitant variation“ (ebd.: 17), was in der autorisierten deutschen Übersetzung mit „correspondenter Ablauf oder Parallelismus der beiderseitigen Erscheinungen“ (Bain 1874 [1872]: 19, Gramm. korrigiert/ M.W.) wiedergegeben wurde.

Vor allem Jackson, Mitherausgeber der ab 1878 erscheinenden Zeitschrift Brain, verbreitete, wie einleitend erwähnt, den Parallelismus in der Folge und verfestigte ihn als Lehre (Senft 1978: 52-58).

The doctrine I hold is: first, that states of consciousness (or, synonymously, states of mind) are utterly different from nervous states; second, that the two things occur together - that for every mental state there is a correlative nervous state; third, that although the two things occur in parallelism, there is no interference of one with the other. This may be called the doctrine of Concomitance. (Jackson 1927 [1884]: 122)

Anders als Bain, der seine philosophiegeschichtlichen Ausführungen zum "essential part of the main argument" rechnete (Bain 1904: 314), betonte Jackson seine Inkompetenz in metaphysischen Fragen. Wie in Deutschland vollzog sich also auch hier in der Rezeption eine Distanzierung von der Philosophie.

Die Betonung der Differenz der beiden Zustände oder Sphären bildete einen Grundzug der britischen Fassung des Parallelismus, der sich ebenso bei Herbert Spencer findet (1885 [1855], Bd. 1: 403)7, auf den sich Jackson in weiten Teilen stützte. Der Parallelismus war für diese Autoren ausdrücklich eine Figur der Bescheidung: Mehr als das zeitliche Zusammengehen könne nicht belegt werden. Besonders prononciert formulierte der Physiker John Tyndall diese Position:

I do not think he [the materialist scientist/M.W.] is entitled to say that his molecular groupings and his molecular motions explain everything. In reality they explain nothing. The utmost he can affirm is the association of two classes of phenomena, of whose real bond of union he is in absolute ignorance. (Tyndall 1871 [1868]: 122)

Eine solche Auslegung des Parallelismus, nach der die Verbindung von Materie und Bewusstsein als unerkennbar angesehen wird, wurde von Thomas Henry Huxley 1869 als Agnostizismus gekennzeichnet (Vidoni 1991: 137) ${ }^{8}$. 
Als Emil Du Bois Reymond 1872 in seiner Leipziger Rede über Die Grenzen des Naturerkennens ähnlich entschieden die Unableitbarkeit des Bewusstseins aus der Materie behauptete wie in England üblich, löste er damit in Deutschland eine heftige Auseinandersetzung, den sogenannten Ignorabimus-Streit aus (Vidoni 1991). Die Verbreitung der agnostischen Position in England und der Sturm, den die Erklärung des "Ignorabimus" [„wir werden (es) nicht wissen"] in Deutschland auslöste, zeigen, wie weit die Auslegungen des Parallelismus auseinander gingen. Gleichwohl wurde der Streit nicht als Auseinandersetzung um die Figur des Parallelismus geführt. Du Bois Reymond hatte sie in seiner Rede nicht gebraucht.

Zeitlich nah an Bains Buch erschien in Frankreich Hippolyte Taines einschlägiges Werk De l'intelligence (1870). Es war ebenfalls inmitten der bereits aufblühenden psychophysischen Forschung entstanden, die der Autor bestens kannte. Gerade weil Taine nicht im Verdacht stand, Materialist zu sein, konnte sein Buch die neue Orientierung in Frankreich maßgeblich verbreiten (Canguilhem 1989 [1980]: 11f.). Taine pflegte gute Kontakte nach Großbritannien und stützte sich ausdrücklich auf den englischen Empirismus Mills und Spencers. Er kannte auch die deutschen Physiologen gut und berief sich außerdem selbstverständlich auf die Pariser Schule der Salpêtrière und den Experimentalismus Claude Bernards. Im engeren Sinne stellte er seinen Ansatz in die Tradition von Condillacs Traité de sensations von 1754. Taine war ansonsten vor allem als Kritiker und Historiker der Literatur hervorgetreten. In De L'intelligence führte er die Forschungen der experimentellen Physiologie mit den Fragen der Erkenntnistheorie zusammen und entwickelte auf dieser Basis seine These von der Wahrnehmung als einer durch Rektifikation entstandenen „wahren Halluzination“. Das Verhältnis von Psyche und Physis steht im Zentrum seines Buchaufbaus: Indem er den psychophysischen Parallelismus in der Buchmitte einführt, markiert Taine mit ihm einen Scheitelpunkt seiner Vorgehensweise (Taine 1880 [1870], Bd. 1: 265). Er eröffnete seine Überlegungen mit einem ausführlichen Zitat Tyndalls, der, wie erwähnt, die Irreduzibilität der „zwei Klassen von Erscheinungen“ betont hatte. Damit nahm Taine den Dualismus der Engländer auf, um ihn jedoch in einen Ansichtsdualismus zu wenden und ihm ähnlich wie Fechner einen ontologischen Monismus zugrunde zu legen:

Wir sind demnach zu der Annahme berechtigt, dass das Gehirnereigniss und das Geistesereigniss im Grunde nur ein und dasselbe, von zwei Seiten betrachtet ist, die eine ist die geistige, die andere die physische, die eine dem Bewußtsein zugänglich, die andere den Sinnen." (Ebd.: 259)

Und weiter: „Jene zwei Seiten sind einander parallel und jede Linie, welche die eine schneidet, schneidet die andere in derselben Höhe." (Ebd.: 263) Tatsächlich war Taine, wie auch Fechner, kein Materialist. Anders als die physiologische Orientierung seines Buches heute glauben machen kann, 
ging er vom Primat des Psychischen aus: „So sind die physischen Ereignisse nur eine rudimentäre Form der psychischen Ereignisse und wir begreifen so den Körper nach dem Modell des Geistes." (Ebd.: 10) Und ebenfalls wie Fechner, auf den er sich aber nicht bezog, griff Taine auf die Schriftmetaphorik zurück, um seinen Parallelismus zu veranschaulichen. Er setzte den Akzent aber entschieden anders, denn er fasste das Verhältnis als eines der Übersetzung (und nicht des Sinns, wie Fechner) auf und verstand dabei die psychischen Ereignisse als Original- beziehungsweise Ursprache, als erste Schrift der Natur:

Denke man sich ein in fremder Sprache geschriebenes Buch, dem eine interlineare Übersetzung beigefügt ist; das Buch ist die Natur, die fremde Sprache ist das psychische Ereignis, die interlineare Übersetzung das physische Ereignis und die Reihenfolge der Capitel ist die Stufenleiter der Wesen. (Ebd.: 264) ${ }^{9}$

Taine markierte von da aus auch die Einseitigkeiten der anderen: „Kurz, die Materialisten leugnen den Text und die Spiritualisten betrachten die Verbindung des Textes mit der Übersetzung als etwas Unbegreifliches. - Wir sind unsere eigenen Wege gegangen." (Ebd.: 264f.) Diese Wege beschrieb er entsprechend als gründliches Studium dieses doppelsprachigen, nicht immer deutlich lesbar geschriebenen Buches der Natur.

De L'intelligence wurde eine wichtige Stütze des französischen Positivismus und prägte die Diskussion in Frankreich nachhaltig. Théodule Ribot, „der so etwas wie Taines Testamentvollstrecker war" (Canguilhem 1989 [1980]: 12), aber auch andere traten das Erbe einer physiologischen Psychologie an, als deren Begründer Taine galt. Wie in Deutschland hat die Rezeption hier Begradigungen und ,Vereindeutigungen' vorgenommen, den idealistischen Standpunkt gestrichen und das Primat der Physiologie behauptet.

\section{Zwischen Psyche und Physis: die Sprache}

Um zu erfassen, wie die Figur des psychophysischen Parallelismus in der konkreten Ausrichtung der Forschung eingesetzt wurde, ist es notwendig, sich für einen Augenblick von ihr zu entfernen und ein ausgesuchtes Diskursfeld zu umreißen. In der zweiten Hälfte des 19. Jahrhunderts liegen sowohl die Anfänge der modernen Aphasiologie als auch die der modernen Linguistik; um beide soll es im Folgenden gehen. Die Sprachforschung beansprucht unser besonderes Interesse, da sie sich nicht ohne weiteres in die Dichotomie von Natur- und Geisteswissenschaften eingliedern lässt. Zudem war der Einsatz des Parallelismus in der Aphasiedebatte besonders klar mit der Forderung nach einer veränderten Forschungsperspektive verknüpft. In der Linguistik tauchte die Figur eher en passant auf, hier wurde aber der Sprache selbst eine bemerkenswerte Stellung innerhalb der Parallelität zugesprochen. 


\section{Die Sprache als Telegraphenapparat}

In der Aphasiologie war der Einsatz des Parallelismus ein kämpferischer. Er verband sich hier mit einer grundsätzlichen Kritik an der Lokalisationslehre. Mit Paul Brocas bahnbrechender Veröffentlichung von 1861 war diese zur Hauptperspektive der Aphasieforscher geworden. Dem Pariser Chirurgen war es durch Sektion gelungen, die Störung des sprachlichen Artikulationsvermögens (motorische Aphasie) in einer Schädigung der dritten linken Frontalwindung des Gehirns zu verorten. Broca beschrieb dabei eine grundsätzliche Teilung des Feldes: Er unterschied die allgemeine Sprachfähigkeit (faculté générale du langage) - die Fähigkeit zur Verknüpfung von Vorstellung und Zeichen, „idée et [...] signe“ (Broca 1861: 334) - von der Fähigkeit zur artikulierten Sprache (faculté de langage articulé). Seine Lokalisation betraf allein die zweite Fähigkeit. Diese von Broca geschaffene Teilung blieb in der nun einsetzenden regen Forschungstätigkeit bestehen, das heißt, das Feld der allgemeinen Sprachfähigkeit wurde tendentiell aus den allgemeinen Lokalisationsbestrebungen ausgeklammert (man erachtete es als zu schwer oder gar nicht lokalisierbar). Die Lokalisationsversuche konzentrierten sich auf die Fähigkeit zur Sprachartikulation, bald auch auf die des Sprachverstehens. Das war der Verdienst des Neurologen und Psychiaters Carl Wernicke, der 1874 der Leistung Brocas die Lokalisation der sensorischen Sprachstörung in der Region des Schläfenlappens an die Seite stellte. Mit dem Postulat der zwei Hauptzentren der Sprachfunktion - eines motorischen (BrocaAreal) und eines sensorischen (Wernicke-Areral) - begründete Wernicke die sogenannte „klassische Lehrmeinung der Aphasie“ (Tesak 2001: 95, Eling 1994) und wurde zum Urheber der „psychophysiologischen Theorie der Sprache" (Jäger 2001: 303).

Das Feld der allgemeinen Sprachfähigkeit wurde mit Wernicke noch entschiedener aus der Aphasieforschung herausgehalten. Er ging hier weiter als seine Vorgänger, indem er den Sprachvorgang grundsätzlich als vom Denkvorgang losgelöst betrachtete: „Denken und Sprechen sind voneinander ganz unabhängige Prozesse“ (Wernicke 1893 [1874]: 31). Damit hatte Wernicke die Unterscheidung von Denkstörung und Aphasie begründet. Aber darüber hinausgehend isolierte er den Sprachvorgang so grundsätzlich vom Denken, dass er ihn als einen „Reflexbogen“ (ebd.: 34) auffassen konnte, in den die Frage des Denkens nicht interveniert. Der Sprachvorgang habe seinen Platz im Hirn an anderer Stelle als das Denken. Über die Störung des Denkens beziehungsweise der „Begriffsregion“, wie es bei ihm heißt, schrieb Wernicke: „Die Sprache an sich braucht darunter nicht zu leiden, wenn auch der Inhalt des Gesprochenen tief blödsinnig ist; der Telegraphenapparat ist in Ordnung, nur das aufgegebene Telegram ist unsinnig." (Ebd.) Wernickes Sprachauffassung hatte sich von der Frage der Verknüpfung von Zeichen und Gedanken oder Vorstellung gänzlich gelöst. Sprache ist bei ihm eine Apparatur, in der Wörter (das sind die sprachlich-psychischen Einheiten, die 
Wernicke betrachtet) übermittelt, aufgenommen und abgegeben werden. Die Wörter selbst liegen im Hirn bereit.

Wernickes Lokalisationstheorie basierte entscheidend auf der Lehre des Hirnanatomen Theodor Meynert. Selbstverständlich betonte er in Absetzung von der verpönten Phrenologie, dass „nur die elementaren psychischen Functionen [...] auf bestimmte Stellen der Großhirnrinde“ (ebd.: 2) verwiesen werden können. Als solche elementaren Funktionen des Sprachvorgangs bestimmte er die sensorischen und motorischen „Erinnerungsbilder" (ebd.) der Wörter, das heißt die Erinnerungen an die Laute (Lautbilder) und Bewegungen (Bewegungsbilder) beim Hören und Sprechen eines Wortes. Seine Vorstellung von der Art und Weise der Lokalisation dieser psychischen Elemente fasste er am Schluss seiner Arbeit in einer sehr konkreten Hypothese zusammen:

Es ist nirgends, so nahe bei dem behandelten Gebiete die Versuchung dazu lag, über die einfachste, wohl kaum noch ernstlich anzufechtende Hypothese hinausgegangen worden, nach welcher dem zentralen Ende jedes Nervenfadens die Rolle eines psychischen Elementes zugeteilt ist. (Ebd.: 66)

Dieser These entsprechend liegt das Psychische am Schlusspunkt einer Nervenbahn, in ihrem letzten Glied. Genauer: Das psychische Element ist in den Rindenkörpern deponiert, die als „Vorratstätten“ (ebd.: 3) der Erinnerungsbilder fungieren. Von solchen Depots ist nach Wernicke die Hirnrinde „bevölkert" (ebd.).

\section{Der Sprachapparat als Assoziationsmechanismus}

An eben dieser Hypothese knüpfte die Kritik der Parallelisten an. Als der entschiedenste Gegenspieler der Lokalisationisten trat der Neurologe Hughlings Jackson hervor, der nicht zufällig zugleich der Hauptverbreiter der Parallelismuslehre in England war. Wenige Jahre nach Brocas Publikation begann er zur Aphasie zu veröffentlichen und blieb von Beginn an der Lokalisation und insbesondere der Annahme von Sprachzentren gegenüber reserviert. „I think, then, that the so-called ,faculty' of language has no existence” (Jackson 1971a [1866]: 123), schrieb er und hielt der Zentrumshypothese eine wesentlich auf evolutionär differenzierten Funktionen aufbauende Theorie der Prozesse entgegen, die hirnorganisch das Sprechen begleiten. Sein Interesse galt weniger den Örtlichkeiten als den neuronalen Prozessen, den Bewegungen. Gestützt auf die Figur des psychophysischen Parallelismus führte Jackson eine für seine Argumentation grundlegende Unterscheidung ein und trennte die Frage der Lokalisation einer (hirnorganischen) Läsion als Ursache einer Sprachstörung von der Lokalisation der Sprachvorgänge als solcher: „To locate the damage which destroys speech and to locate speech are two different things." (Jackson 1971b [1874]: 130) Die Sprache oder das Sprechen lokalisieren zu wollen, beruhe auf einer Verwechslung der beiden Sphären: 
We must as carefully distinguish betwixt words and their physical basis, as we do betwixt colour and its physical basis; a psychical state is always accompanied by a physical state, but nevertheless the two things have distinct natures. (Jackson 1971c [1878]: 160)

Diese Trennung war für Jackson entscheidend und er bestand penibel auf der sprachlichen Formulierung. Weiter heißt es:

\begin{abstract}
Hence we must not say that the memory of words' is a function of any part of the nervous system, for function is a physiological term (vide infra). Memory or any psychical state arises during not from - if 'from' implies continuity of a psychical state with a physical state - functioning of nervous arrangements, which functioning is a purely physical thing - a discharge of nervous elements representing some impressions and movements. Hence it is not to be inferred from the rough division we just made of the elements of 'talking', and from what was said of their 'separation' by disease, that there is anything in common even for reasonable contrast, much less for comparison, betwixt loss of speech (a psychical loss) and immobility of the articulatory muscles from, say disease of the medulla oblongata, as in 'bulbar paralysis' (a physical loss). (Ebd., Hervorh. i. Original)
\end{abstract}

Schließlich mündet Jacksons Sprachkritik in die Warnung, die Methode, die eine Gegenstandsklasse erfordere, wohl zu beachten:

As before said, we must not classify on a mixed method of anatomy, physiology and psychology, any more then we should classify plants on a mixed natural and empirical method as exogens, kitchen herbs, graminaceæ and shrubs. (Ebd.)

Die Zusammenstellung hat Züge der berühmten chinesischen Enzyklopädie von Borges, die Foucault in Die Ordnung der Dinge (1974 [1966]: 17) zitiert, um aus der Verrücktheit ihrer Klassifizierung heraus die Frage zu entwickeln, was denn überhaupt die verschiedenen Ordnungen des Wissens reguliere, die es in der Geschichte gegeben hat. Es sei daran erinnert, dass Jackson sich tatsächlich inmitten eines Geschehens äußert, das von einer Um- und Neuordnung des Wissens gezeichnet war. Die aufgeführten Fächer rangen um ihre Konturen und suchten sich im Feld der Wissenschaften möglichst stark zu positionieren. Jackson trat mit der Parallelismuslehre für eine Neurophysiologie ein, nach der in erster Linie Prozesse und deren Zusammenhänge betrachtet werden sollten, denn er sah das Nervensystem als Bewegungsorgan an und seinen Aufbau - im Anschluss an Spencer - als eine evolutionär begründete Funktionshierarchie. Seine Aufmerksamkeit für das Prozesshafte hat ihn dabei auch die Sprache als Verkettung, also als einen Assoziationsmechanismus begreifen lassen. Das war eine Position, die unter den damaligen Aphasiologen kaum verbreitet war. Für Jackson war Sprache nicht ein Haufen von Wörtern, „a word-heap”, wie er polemisch formulierte. „Speaking is not simply the utterance of words. The utterance of any number of words would not constitute speech. Speaking is ,propositionizing." (Jackson 1971b [1874]: 130) Jackson setzte sich mit dem Parallelismus für eine neue Ausrichtung der Neurologie ein. Seine Arbeiten fanden seinerzeit allerdings wenig Verbreitung. Sie galten, auch wegen Jacksons eigenwilliger sprachlicher Penibilität, als schwer lesbar und blieben in der damaligen Aphasie-Debatte 
ohne durchschlagende Resonanz. Aus heutiger Sicht erscheinen sie modern, und tatsächlich wurden sie bereits Anfang des 20. Jahrhunderts insbesondere im Zuge der Herausbildung holistischer Ansätze wiederentdeckt und verstärkt rezipiert (Tesak 2001: 124, Leuschner 1992: 19).

Ein weiterer Kritiker des Lokalisationismus, der sich ebenfalls auf die Figur des Parallelismus stützte, war der junge Sigmund Freud. So sehr sich die Grundrichtung seines Interesses von dem Jacksons unterschied, so nah schloss er sich doch an dessen Perspektive in dieser Sache an. Der Wiener Neurologe bildete tatsächlich eine Ausnahme unter den frühen Lesern Jacksons. Wie dieser stellte sich Freud in seiner Aphasiearbeit gegen die lokalisatorische Theorie der Sprachstörungen und ihre Annahme von Zentren und forderte ebenso eine grundsätzliche Perspektivwende der Aphasieforschung, die die „Funktionsbedingungen des Sprachapparates“"10 (Freud 1992 [1891]: 149) und dessen „Natur als Assoziationsmechanismus“ (ebd.: 148f.) in den Vordergrund stellen sollte. Wie Jackson widersprach Freud Wernickes Versuch, Sprache und Denken voneinander zu lösen (ebd.: 121f., Jackson 1927 [1884]: 92). Und er stützte sich in seiner „kritischen Studie“ auf den psychophysischen Parallelismus in Jacksons Formulierung:

Die Kette der physiologischen Vorgänge im Nervensystem steht ja wahrscheinlich nicht im Verhältnis der Kausalität zu den psychischen Vorgängen. Die physiologischen Vorgänge hören nicht auf, sobald die psychischen begonnen haben, vielmehr geht die physiologische Kette weiter, nur daß jedem Glied derselben (oder einzelnen Gliedern) von einem gewissen Moment an ein psychisches Phänomen entspricht. Das Psychische ist somit ein Parallelvorgang des Physiologischen (,a dependent concomitand'). (Freud 1992 [1891]: 98)

Freud wandte sich mit diesen Sätzen direkt gegen das Modell Wernickes, welches, wie er sagte, „Vorstellungen in Zellen bannt“ (ebd.: 97) - nämlich die Worterinnerungsbilder, wie oben beschrieben, an das Ende bestimmter Nervenfasern -, und hielt diesem das Hauptargument des Parallelismus entgegen: die Geschlossenheit der Naturkausalität.

Jackson und Freud gebrauchten die Figur des psychophysischen Parallelismus beide als Argument für die Differenz der Sphären und für die Notwendigkeit gesonderter Herangehensweisen. Sie setzten die Figur polemisch ein gegen die Theorie der Lokalisationisten, und erst in zweiter Linie als Stützte für den eigenen Entwurf. In ihren weiteren Arbeiten gaben sie der Formel allerdings einen sehr unterschiedlichen Stellenwert. Für Jackson blieb sie von grundlegender Bedeutung. Als Neurologe und Aphasiologe (er schrieb allein 28 Texte zur Aphasie) baute er darauf, denn sie sicherte seinen Forschungen den Rahmen einer geschlossenen Kausalität. „Er sagte einmal, daß er, falls er von einem sich gegenseitig beeinflussenden Dualismus überzeugt würde, die Erforschung des Nervensystems aufgäbe." (Mercier zit. nach Senft 1978: 58) Freud dagegen verfasste nur diese eine Studie zur Aphasie und steuerte auf eine Theorie des Psychischen zu, die sich von den Gesetzen der Neuroanatomie und Physiologie löste. Für ihn hatte die Figur des psychophy- 
sischen Parallelismus die Funktion gehabt, die beiden Sphären voneinander zu scheiden und dem psychischen Raum eine eigene Gesetzlichkeit zuzusprechen, ohne das Feld der Wissenschaften zu verlassen. Freud griff auf die Figur später nicht wieder zurück. Er konnte sich sogar in einem anderen, fast zeitgleich entstandenen Text positiv auf die Wechselwirkung beziehen. „Die ärztliche Wissenschaft hatte hier“, so schrieb er 1890 über die Neurotiker, die an einem „veränderten Einfluß ihres Seelenlebens auf ihren Körper“ leiden, „die Anknüpfung gefunden, um der bisher vernachlässigten Seite in der Wechselbeziehung zwischen Leib und Seele ihre Aufmerksamkeit im vollen Maß zuzuwenden." (Freud 1982 [1890]: 20) Als die Psychoanalyse geschaffen und theoretisch ausgebaut war, verabschiedete Freud sich ausdrücklich vom Parallelismus und seinen „unlösbaren Schwierigkeiten“ (Freud 1999 [1915]: 266, vgl. Wegener 2004: 167). Er nahm aber auch die Figur der Wechselwirkung nicht zu seinem neuen Bezugspunkt. Vielmehr sprach Freud dem Grundkonzept seiner Lehre, dem Unbewussten, selbst den Platz zwischen Psyche und Physis zu und bezeichnete es als „die richtige Vermittlung zwischen dem Körperlichen und dem Seelischen, vielleicht das langentbehrte ,missing link“" (Freud/Groddeck 1988: 15). Man kann vermuten, dass die hybride epistemologische Position, die sich die Psychoanalyse seither eingehandelt und bis heute innehat, mit dieser Antwort zusammenhängt. Immerhin hat sie sich als Wissenschaft des Unbewussten genau an die Nahtstelle gesetzt, an der seit der Zweiteilung Natur- und Geisteswissenschaften um ihre Gebietshoheit ringen.

\section{Die Chemie der Sprache}

Einen weniger polemischen Einsatz als in der Aphasieforschung fand die Figur des psychophysischen Parallelismus in der Begründung der modernen Linguistik. Hier kam sie nicht kämpferisch, nicht einmal emphatisch zum Einsatz, wurde aber in besonders enger Weise mit dem Gegenstand der Linguistik - der Sprache - selbst verknüpft. Der Schweizer Sprachwissenschaftler Ferdinand de Saussure griff in seiner Neubegründung des Fachs auf das Parallelenbild in Zusammenhang mit der Zwischenstellung der Sprache zwischen Körper und Seele zurück.

Wie Ludwig Jäger herausgearbeitet hat, bezog sich Saussure in seiner Neudefinition des Gegenstands der Sprachwissenschaft durchaus auf die Arbeiten der Aphasiologen. Dabei nahm er insbesondere Brocas Unterscheidung von sprachlicher Artikulation und allgemeiner Sprachfähigkeit auf (Jäger 2001: 305f.) - und wandte sich genau der Seite dieser Zweiteilung $\mathrm{zu}$, die von der zeitgenössischen Aphasieforschung ausgeblendet worden war. Die Broca'sche Zweiteilung kehrt bei Saussure in Form der Unterscheidung von Sprechen (parole) und Sprache (langue) wieder, und das Primat liegt nun ganz auf der Sprache: „Alle anderen Elemente der menschlichen Rede, die das Sprechen ausmachen, ordnen sich von selber dieser ersteren 
Wissenschaft [von der Sprache, M.W.] unter." (Saussure 1967 [1916]: 21) Die allgemeine Sprachfähigkeit besteht für Saussure wie für Broca in einer Verknüpfungsleistung, dem „centre associatif“, wie Saussure es nennt (Jäger 2001: 316). Aus dieser Verknüpfung geht nach Saussure das Zeichen hervor, welches das zusammengesetzte Grundelement der Sprache bildet. Das Zeichen und die Zeichenbeziehungen sind die „konkreten Tatschen“ oder „wirklichen Objekte“ der Sprachwissenschaft (Saussure 1967 [1916]: 122). Das Zeichen entsteht - für diese Definition wurde Saussure berühmt - aus der Zusammenfügung von Lautbild und Vorstellung, die dann, im Zeichen, als Verknüpfung von Signifikant und Signifikat auftritt. Hier, im doppelten Antlitz des Zeichens, kommt das Verhältnis von Körper und Seele ins Spiel.

Saussure hält fest, dass der Laut für sich genommen ein Gegenstand der Physiologie ist, während die Vorstellung, der Begriff oder der Gedanke (in Saussures Texten finden sich alle drei Termini) psychologische Gegenstände sind. Mit dem Zeichen, dem „nœud psychique“ (Saussure zit. n. Jäger 2001: 318), wird, wie er betont, etwas gebildet, das mehr als eine einfache Verbindung beider Seiten ist, etwas wirklich Neues:

\footnotetext{
Man hat diese mit doppeltem Antlitz ausgestattete Einheit mit der Einheit des Menschen, die aus Körper und Seele zusammengesetzt ist, verglichen. Dieser Vergleich ist jedoch nicht befriedigend. Man könnte richtiger an einen chemischen Körper denken, das Wasser z.B.; es ist die Verbindung von Wasserstoff und Sauerstoff; jedes der Elemente hat, für sich genommen, keine der Eigenschaften des Wassers. (Saussure 1967 [1916]: 123)
}

Bevor die Verbindung entsteht, die das Zeichen konstituiert, sind beide Entitäten - die Laute wie das Denken - „gestaltlose und unbestimmte Massen[n]“ (ebd.: 133). Saussure betonte: „Philosophen und Sprachforscher waren immer darüber einig, daß ohne Hilfe der Zeichen wir außerstande wären, zwei Vorstellungen dauernd und klar getrennt zu halten. Das Denken für sich genommen ist wie eine Nebelwolke, in der nichts notwendigerweise begrenzt ist.“ (Ebd.) Und er fuhr fort: „Die lautliche Masse ist ebensowenig etwas fest Abgegrenztes und klar Bestimmtes." (Ebd.) Statt einer einfachen Verbindung dieser beiden „Massen“ bringt das Dazwischentreten der Sprache hier völlig neue Eigenschaften hervor - es bewirkt gleichsam eine chemische Verwandlung der Teile und erschafft so etwas Drittes, Neues. Die Sprache trägt Differenz ein, wo vorher keine war. Die berühmt gewordene Darstellung, die der Cours de linguistique überliefert, lässt „das unbestimmte Feld der vagen Vorstellungen (A)“ und das „ebenso unbestimmte Gebiet der Laute (B)“ wie Wolken über Wasser erscheinen (Abb. 1).

Zwischen sie tritt die Sprache als „milieu intermédiaire“ (Saussure zit. n. Jäger 2001: 329) und bringt auf beiden Seiten Artikulation hervor.

[E]s findet also weder eine Verstofflichung der Gedanken noch eine Vergeistigung der Laute statt, sondern es handelt sich um die einigermaßen mysteriöse Tatsache, daß der ,Laut-Gedanke' Einteilungen mit sich bringt, und die Sprache ihre Einheiten herausarbeitet, indem sie sich zwischen zwei gestaltlosen Massen bildet. (Saussure 1967 ]1916]: 134) 


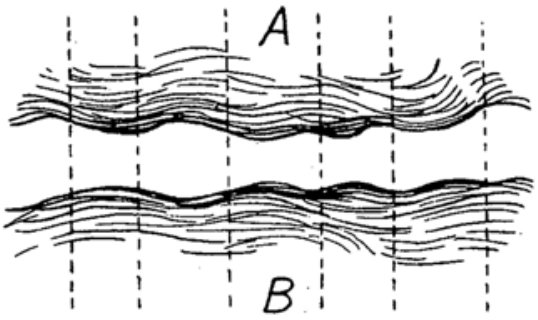

Es handelt sich nicht um präexistente Entitäten, vielmehr bilden sich unterscheidbare Gedanken und lautliche Elemente erst durch die Intervention der Sprache. Genau hier wird im Cours die Figur der Parallelen herangezogen, die bereits in der Zeichnung aufscheint. Die Einteilungen, die das Dazwischentreten der Sprache hervorbringt, bewirken, dass aus dem Laut- und Gedankenstrom Signifikanten und Signifikate werden. Jede Reihe ist „für sich genommen lediglich differentiell und negativ“ (ebd.: 144), heißt es hier, wobei „negativ“ besagt, dass die Elemente der Reihe allein durch ihre Unterschiedenheit, nicht durch irgendein positives Merkmal bestimmt sind. Erst in der von der Sprache hergestellten Verbindung entsteht nach Saussure ein positives Faktum, eine Positivität. Und, wie im Cours betont wird, ist „gerade dies das besondere Wesen der Sprache [...], daß sie den Parallelismus zwischen diesen beiden Arten von Verschiedenheiten aufrecht erhält" (ebd.: 144). ${ }^{11}$

Die Figur des Parallelismus erfährt hier eine beachtenswerte Wendung. Sie fungiert in der Linguistik nicht als eine Formel für das Verhältnis von Körper und Seele als solches, sondern bezeichnet einen Zusammenhang, der erst hergestellt werden muss. Die Figur verdankt ihren Stand dem Dazwischentreten der Sprache. Denn erst die Sprache artikuliert die zwei „Arten von Verschiedenheiten“ in einer Weise, die parallel genannt werden kann. Damit kennzeichnete Saussure die Sprache auch in diesem Feld als primär, das heißt dem Verhältnis von Körper und Psyche vorgängig. Er fügte zugleich an dieser Nahtstelle eine überindividuelle Dimension ein, denn die Sprache ist, wie er verschiedentlich betonte, eine irreduzibel soziale Gegebenheit. Zugleich brachte Saussure in der Bezugnahme auf die Figur des psychophysischen Parallelismus seine Wissenschaft in Stellung: Die Linguistik brauche nicht abzuwarten, bis eine korrekte Formulierung des Körper-Seele-Verhältnisses geschaffen ist. Die Sprache - obwohl sie mit den beiden Seiten dieses Verhältnisses intim verknüpft ist - könne und müsse unabhängig von einer solchen Formulierung definiert und erforscht werden. Damit erlangte die Linguistik Eigenständigkeit gegenüber den Natur- und Geisteswissenshaften und eine gewisse Freiheit gegenüber deren Auseinandersetzungen (ebd.:
Abb. 1: Die Sprache tritt zwischen Denknebel und Lautstrom (Saussure 1967 [1916]: 133). 
7f.). Es wurde vielmehr denkbar, dass eine genauere Kenntnis der Sprache dazu beitragen könnte, die Verknüpfung von physiologischen und psychologischen Elementen aufzuklären und sich der „einigermaßen mysteriöse[n] Tatsache“ zu nähern, wie diese Verknüpfung beide Seiten verändert.

\section{Der Fall der Figur des psychophysischen Parallelismus}

\section{Auflösung der Vormachtstellung}

Zurück zu den großen Debatten um den Parallelismus: Der Moment, in dem die Figur erstmals wirklich prominent platziert wurde - 1894 mit Wundts Titel und 1895 mit einem Lexikoneintrag im Brockhaus - fiel zusammen mit dem ersten Verlust ihrer Selbstverständlichkeit. Wundts Text Ueber psychische Causaliät und das Princip des psychophysischen Parallelismus, der zunächst als eine umfangreiche philosophische Reflexion daherkommt, ist genau besehen eine Streitschrift. Wundt versuchte hier die Figur des psychophysischen Parallelismus einer falschen Interpretation zu entreißen, um sie einer richtigen, „empirischen“ (Wundt 1894: 34) zuzuführen. Sie wurde in diesem Text Gegenstand eines offen geführten Streits um die Deutungshoheit. Wundt, der sich seit 1862 zum Parallelismus bekannte (Heidelberger 2000: 59), zielte darauf ab, die hoch im Kurs stehende Figur aus dem vorherrschenden naturwissenschaftlich-materialistischen Kontext, in dem sie in Deutschland stand, herauszulösen und für die Etablierung der Psychologie als Geisteswissenschaft fruchtbar zu machen. Der Begründer der experimentellen Psychologie in Deutschland distanzierte sich, auch gegen seine Schüler, ausdrücklich von der "materialistischen Psychologie" (Wundt 1894: 46f.) und betonte die Verwandtschaft der Psychologie mit der Philosophie statt mit den Naturwissenschaften. Schon den Titel seiner Zeitschrift Philosophische Studien, in der der genannte Aufsatz erschien, verstand er in diesem Sinne als „Kampfestitel“ (Wundt zit. n. Schmidt 1995: 83).

Diese Ereignisse standen inmitten der Trennungsgeschichte von Naturund Geisteswissenschaften und bildeten einen der Höhepunkte der Auseinandersetzung. Im selben Jahr publizierte Wilhelm Dilthey seine berühmte und trennungsgeschichtlich einschlägige - weil eine gesalzene Replik von Ebbinghaus provozierende - Akademieabhandlung Ideen über eine beschreibende und zergliedernde Psychologie, mit der er die Psychologie zum Entscheidungsort des Streites erklärte (ebd.: 37f., Ash 1999, 2007). In dem Bestreben, den Geisteswissenschaften ein eigenständiges Fundament zu geben, standen Dilthey und Wundt auf derselben Seite. Beide sprachen der Psychologie dabei eine zentrale Rolle zu, da ihr Gegenstand durch seine unmittelbare Zugänglichkeit im bewussten Erleben gekennzeichnet sei, was auf die Gegenstände der Naturwissenschaften, die immer mittelbar gewonnen werden, nicht zutreffe (Dilthey 1974 [1894]: 143f.). Dilthey, der einige „be- 
freundete Psychologen und auf dem Gebiet der Psychologie arbeitende bzw. psychologisch interessierte Philosophen" um konstruktive Kritik gebeten hatte (Schmidt 1995: 43), erhielt durch Wundt mit dem genannten Aufsatz Schützenhilfe. Wundt griff darin insbesondere seinen ungetreuen Schüler Hugo Münsterberg und Theodor Ziehen für ihre "metaphysische“ (Wundt 1894: 26) und, wie er meinte, spinozistische Auslegung des Parallelismus scharf an, die die „Seele als einen geistigen Spiegel für den ihr zugänglichen Inhalt der objektiven Welt" (ebd.) ansehe und diese so zu einem bloßen Abbild der Naturvorgänge degradiere. Eine so verstandene Psychologie würde die von Wundt erstrebte Selbstständigkeit des Faches als Geisteswissenschaft verfehlen. Wundts Hauptanliegen bestand daher in der Etablierung seines Begriffs der „psychischen Kausalität“, mit dem er eine Gesetzlichkeit festzuhalten suchte, auf der sich die Eigenständigkeit der psychologischen Wissenschaft begründen ließe. Seine Argumentation entwickelte er dabei nicht in Auseinandersetzung mit J. St. Mill, sondern vielmehr mit der 1873-78 erschienenen Logik Christoph Sigwarts. In seiner Auslegung des Parallelismus ging Wundt von zwei unabhängigen, in sich geschlossenen Kausalreihen aus, ohne auf die hierin verwandte britische Variante Bezug zu nehmen. Mit einem überraschenden panpsychistischen Schlenker endend, betonte er:

Die geistigen Vorgänge sind nicht mehr Abbilder der physischen Vorgänge, sondern von ihnen toto genere verschieden, und sie stehen mit ihnen nur in dem Sinne in einer festen Beziehung, dass jedem materiellen Vorgang ein geistiger und jedem geistigen Vorgang ein materieller entspricht. (Ebd.: 41)

Fechner war noch nah.

Die größte Anstrengung bezüglich des Parallelismus unternahm Wundt in dem Artikel allerdings mit seiner Umdeutung der Figur. Er vollbrachte das Kunststück, sie mit eben jener Auffassung zu versöhnen, gegen die sie Mitte des Jahrhunderts angetreten war: der Vorstellung einer Wechselwirkung zwischen Psyche und Physis. Gerade die Verabschiedung der Wechselwirkung hatte dem Parallelismus aber seine deutlichste Kontur verliehen. Daher brachte die Neudeutung ihn so grundlegend ins Schwanken, dass die Figur gekippt wäre, wenn Wundt nicht gleichzeitig so überzeugt an ihr festgehalten hätte. Für ihn besaß sie einen so hohen wissenschaftlichen Wert, dass er keinen argumentativen Aufwand scheute, sie für sich zu übersetzen. Die Figur des Parallelismus war für ihn die wissenschaftlich korrekte Formulierung, die er als die „unmittelbare Folgerung“ (ebd.: 38) aus dem Prinzip der geschlossenen Naturkausalität anerkannte. Gleichwohl war es für Wundt entscheidend, der „populären Bedeutung“ und der „erfahrungsgemäß“ (ebd.: 27) verankerten Wechselwirkung von Psyche und Physis eine exakte Formulierung zu geben und damit den wissenschaftlichen Zugriff der Psychologie zu erweitern und zu stabilisieren. Wundt nahm, abgekürzt gesprochen, hierfür den Weg, die Vereinbarkeit der Figur der Wechselwirkung mit dem Prinzip der geschlossenen Naturkausalität aufzuweisen. Dazu entwickelte er 
eine Definition der Wechselwirkung, bei der die Gesetzmäßigkeit der Physis völlig ungestört und so geschlossen blieb.

Nichts steht nämlich im Wege zu sagen: Psychische Effecte physischer Ursachen sind psychische Vorgänge, die aus einer physischen Causalreihe derart hervorgehen, dass ihre Entstehung in dem Ablauf jener physischen Reihe keine Veränderung hervorbringt; und physische Effecte psychischer Ursachen sind physische Vorgänge, die mit psychischen Bedingungen regelmäßig verknüpft sind, dabei aber physisch betrachtet immer auch aus einer physischen Causalreihe vollständig ableitbar seien müssen. (Wundt 1894: 36)

Mit seiner gewundenen Argumentation setzte er schließlich die Differenz zwischen Parallelismus und Wechselwirkung aus und kam zu dem Schluss: „Unter dieser Voraussetzung ist das Parallelprincip der einfachste Ausdruck der Thatsachen der psychophysischen Wechselbeziehungen." (Ebd.: 38) Damit war aber auch die Absetzung gelöscht, aus der die Figur seit Mitte des Jahrhunderts ihre Kontur und ihre Kraft bezogen hatte.

In dem Augenblick, in dem die Vorstellung auftauchte, dass die als unwissenschaftlich verpönte Wechselwirkung selbst auf dem Boden der Wissenschaft formuliert werden könnte, wurde sie zur ebenbürtigen Formel. Darauf setzte entweder der Versuch ein, die Differenz zwischen der neuen und der alten Figur zu nivellieren wie Wundt dies tat, oder es brach erneut der Streit um die Figuren aus, in den die alte Figur nun als neu erstarkte Konkurrenz eintrat. Letzteres war der Fall in der nun aufziehenden Diskussion.

\section{Der Auftakt des Parallelismusstreits}

Zwei Jahre später, im August 1896, griff der Experimentalpsychologe Carl Stumpf in seiner Eröffnungsrede auf dem dritten internationalen Kongress für Psychologie in München die „Parallelitätslehre“ (Stumpf 1897: 9) nun tatsächlich frontal an. Der an prominentem Ort und in grundsätzlicher Weise geführte Schlag bildete den Auftakt des „Parallelitätsstreits“ (Busse 1903: 2), aus dem zahlreiche Publikationen hervorgingen. Nicht nur Stumpf war sensibilisiert für das Thema, aber er war es wohl in besonderem Maße. Wesentlich auf Betreiben Diltheys war er 1893 auf den neu eingerichteten Lehrstuhl für experimentelle Psychologie am philosophischen Institut in Berlin berufen worden und hatte Publikation und Wirkung von Diltheys Grundsatzthesen zur Psychologie daher aus nächster Nähe verfolgen können. Dilthey hatte sich nachdrücklich für Stumpfs Berufung eingesetzt, um Unterstützung für die geisteswissenschaftliche Orientierung am Institut zu gewinnen (Siegert 1999: 180). Als er dem offenbar zunächst unwilligen Stumpf endlich die Zusage abgerungen hatte, triumphierte Dilthey in einem Brief an York von Wartenburg, „mein Eingreifen hat gänzliche naturwissenschaftliche Radicalisierung der Philosophie hier verhütet“ (Dilthey zit. n. Siegert 1999: 181). Damit war der erste Lehrstuhl des zukünftigen psychologischen Instituts in Berlin geschaffen. Als es im Jahr 1900 aus dem Psychologischen Seminar entstand, war es eines der ersten selbstständigen Psychologischen Institute 
an der Universität überhaupt. ${ }^{12}$ Später gingen aus Stumpfs Lehre die Begründer der Berliner gestaltpsychologischen Schule hervor.

Stumpfs Stellungnahme zum Parallelismus fiel wesentlich kürzer aus als der Beitrag Wundts zur Debatte, dafür aber entschieden pointierter. Er hatte für sie eine öffentlich exponierte Position vor bestmöglichem Publikum gewählt. Die seit 1889 stattfindenden Psychologenkongresse waren zu einem großen internationalen Forum der physiologisch und experimentell ausgerichteten Psychologie geworden, das die führenden Köpfe des Fachs versammelte. ${ }^{13}$ Nach den Präliminarien gab Stumpf „die Function des Sprachrohrs" (Stumpf 1897: 8) auf, die ihm als Präsident und Eröffnungsredner zukam, und kündigte einen „Eigenton“ (ebd.) an, den er im Folgenden nicht unterdrücken könne. Im Unterschied zu Wundt ließ Stumpf die Figur des Parallelismus nun öffentlich fallen. Nach seinen Ausführungen ist sie völlig ungeeignet, das Zusammenspiel psychischer und physischer Vorgänge zu erfassen, das ihn gerade interessierte. Um die Figur zu entthronen griff er als erstes den guten Ruf an, den sie besonders in Deutschland als Überwinderin des Dualismus genoss: „[I]ch kann in der Parallelitätslehre überhaupt statt des gepriesenen Monismus nur einen Dualismus finden, wie er crasser noch niemals aufgetreten ist." (Ebd.: 10) Die Formel betone die Heterogenität der Gebiete, zwischen denen jegliche Interaktion negiert werde. Auch die Rede von der einheitlichen Substanz (etwa bei Fechner) könne diese Trennung kaum überzeugend überbrücken, denn sie sei nichts weiter als ein metaphysischer "Hilfsbehelf" (ebd.). Es sei, so Stumpf, keine Auslegung des Parallelismus zu finden, die diesem Manko entkäme. Grundsätzlich unterschied er zwei Varianten: Entweder würde allein den physischen Vorgängen eine Kausalität zugesprochen, wodurch die psychischen Vorgänge zu reinen "Schatten- oder Spiegelbilder[n]“ (ebd.: 9) derselben verkommen (Wundts Kritik), oder es würden zwei eigenständige Kausalitäten anerkannt (Wundts Lösung). In diesem Fall sei aber die Konsequenz des „Panpsychismus“ (ebd.: 10) unentrinnbar und der Dualismus werde zementiert.

Stumpf, den die Frage des Parallelismus im Übrigen nicht loslassen sollte (Stumpf 1919), sprach sich 1896 explizit für die Figur der Wechselwirkung aus, das heißt für eine Kausalität zwischen Physis und Psyche. Er formulierte aber auch jenseits aller Gegenüberstellungslogik: Es gehe ihm darum, das Psychische „ohne Verletzung des Energiegesetzes in den allgemeinen Causalzusammenhang einzufügen" (Stumpf 1897: 12 ${ }^{14}$ ). Stumpf machte zwei Vorschläge, wie die Annahme von Kausalvorgängen zwischen Psyche und Physis mit dem Gesetz von der Erhaltung der Energie - der stärksten Stütze des Parallelismus - zu vereinen sei. Sein erster Vorschlag ging von der Existenz einer eigenen ,psychischen Energie‘ aus. Es „ließe sich das Psychische ganz wohl als eine Anhäufung von Energien eigener Art ansehen, die ihr genaues mechanisches Äquivalent hätten“ (ebd.: 12), hielt er fest und knüpfte daran den Gedanken, dass sich diese psychische Kraft dann als eine mög- 
liche Energieform unter anderen in die allgemeinen Transformationsvorgänge einbeziehen ließe. ${ }^{15}$ Als Alternative stehe aber - sein zweiter Vorschlag - auch eine Konzeption offen, nach der die psychischen Folgen beziehungsweise Ursachen physischer Vorgänge ohne energetische Eingriffe in die letzteren denkbar seien. Hier würde aus einem bestimmten Nervenprozess eine bestimmte Empfindung "als notwendige Folge neben den physischen Wirkungen" hervorgehen (ebd.: 13), ohne dass es zu einem Austausch von Kräften käme. Wenn ein Parallelist hierin seine Auffassung wiedererkennen sollte, tue er dies, wie Stumpf mit einem Seitenhieb gegen Wundt bemerkte, „inconsequenter Weise“ (ebd.). Mit einer solchen Auffassung sei der parallelistische Standpunkt bereits verlassen.

Stumpf eröffnete mit seiner Rede die Diskussion um die Figur des Parallelismus auf grundsätzlicher Ebene, sie enthielt wesentliche, in den folgenden Debatten beständig wiederkehrende Koordinaten. Zu diesen gehörten: die Gegenüberstellung der Figur des Parallelismus und der der Wechselwirkung, die man nun neu zu deuten versuchte, die Auseinandersetzung mit dem Krafterhaltungssatz und der Geschlossenheit der Naturkausalität und grundsätzlich die erneute Befragung des Kausalitätsbegriffes, die zunehmend ins Zentrum rückte. Alle diese Elemente waren im Einzelnen nicht neu, wurden jetzt aber neu in Stellung gebracht.

Auf dem Kongress von 1896 fand Stumpfs Angriff noch keinen Widerhall in der Diskussion um die Figur des psychophysischen Parallelismus, obwohl hier heftig um die Ausrichtung der Psychologie im wissenschaftlichen Feld zwischen Physiologie und Philosophie gestritten wurde. So forderte Bechterew in einer lebhaften Auseinandersetzung, die der Vortrag von Paul Flechsig ausgelöst hatte, „dass der künftige IV. Congress sich schon als rein physiologischer darstelle“, woraufhin sich Stumpf veranlasst sah, die Diskussion als „fruchtlos“ zu schließen (Anonym 1897: 73, vgl. 225-230, 284287). Der Parallelismusstreit brach erst einige Jahre später, in Deutschland vor allem in der Zeitschrift für Philosophie und Philosophische Kritik und in verschiedenen größeren Publikationen, aus. Die Figur des Parallelismus rangierte jetzt nicht mehr in Einleitung oder Schlusskapitel konkreter Untersuchungen, sondern wurde Gegenstand eigenständiger Abhandlungen. Das veränderte ihren Status weitreichend: Die Figur trat nicht mehr als Markierung eines naturwissenschaftlichen Textes auf, dessen Stellung in Grundsatzfragen sie kenntlich machte, wobei sich die Autoren mehr oder weniger weit in erkenntnistheoretisches Gebiet vorwagten. Sie wurde umgekehrt im Feld der Erkenntnistheorie und Logik selbst befragt und verschwand tendenziell aus den Arbeiten der Forscher. Wundts und Stumpfs Texte standen am Übergang des alten und des neuen Gebrauchs der Figur. Es waren nicht zufällig Psychologen, die die Diskussion anstießen, denn ihr Fach - noch nicht lange emanzipiert von der Philosophie und in nicht ganz geklärter Nähe zur Neurophysiologie - lag im Schnittpunkt der Fragen. Die Autoren, die in der 
Folge die Bühne betraten, waren fast ausschließlich Philosophen. Dabei war man stolz gewesen, die Figur aus der rein philosophischen Diskussion herausgelöst und mit empirischer Forschung verknüpft zu haben. Jetzt wurde sie von der Philosophie zurückerobert. Dies geschah in dem Moment, da in der Etablierung der neuen Fächer ein ausreichend stabiler Status erreicht und die Durchsetzung der Physikalisierung unumkehrbar war. Die Figur des Parallelismus hatte diesen Wandel mitgetragen und hatte, solange sie diese tragende Funktion innehatte, nicht selbst Gegenstand der Befragung werden können. Dies geschah jetzt mit Nachdruck.

\section{Die Philosophie übernimmt}

An den nun einsetzenden regen Diskussionen beteiligten sich zahlreiche bekannte und unbekanntere Autoren. Die berühmteste Stimme kam aus Frankreich: Henri Bergson wurde zum wichtigsten Kontrahenten Hippolyte Taines (Brunschvicg 1931: 33, Canguilhem 1989 [1980]: 12). Er widmete sich 1904 in einer Rede explizit dem psychophysischen Parallelismus und unterzog die Figur, über deren „durchaus metaphysische Ursprünge“ (Bergson 1928 [1904]: 171) er keinen Zweifel ließ, einer rigoros philosophischen Kritik. Bergsons Vorgehen unterschied sich, wie später noch deutlicher werden wird, im Ansatz von dem der Kritiker in Deutschland, denn er stellte unmissverständlich klar, dass die Figur im Register der Metaphysik zu diskutieren sei. Seinen Ausgangspunkt bildeten die zwei großen philosophischen "Notierungssysteme“ des Wirklichen (ebd.: 171), Idealismus und Realismus. Bergson zielte auf den Nachweis einer inneren Unlogik des Parallelismus, den er als einem falschen Kompromiss zwischen diesen beiden Systemen entlarvte: In der „scheinbaren Versöhnung zweier unvereinbarer Auffassungen liegt das Wesen der Parallelismus-Theorie“ (ebd.: 185). Für ihn gab diese Idee „den Grundton“ (ebd.: 188) eines ganzen Akkords von Folgeirrtümern an, als die er aufzählte: die Idee einer zerebralen Seele, die mechanistische Kausalitätsvorstellung, die Abwertung der Bilder gegenüber der Abstraktion und den Fehlschluss von der Solidarität der Gesamtheit auf die Äquivalenz der Teile. Bergson hielt fest: „Sie alle zusammen bilden jetzt um sie her eine imposante Verteidigungslinie, die man nicht an einem Punkte durchbrechen kann, ohne dass sich der Widerstand an einem anderen erneuert." (Ebd.: 185). Gleichwohl habe er „versucht, diese Täuschung zu vernichten" (ebd.). Bereits in seinem Hauptwerk Matière et Memoire (1896) hatte Bergson das Verhältnis von Leib und Psyche ins Zentrum gesetzt, er stand mit der ganzen Emphase seines Philosophierens gegen den Mechanismus des 19. Jahrhunderts, in dessen Regime er der Figur des Parallelismus einen zentralen Platz zuwies.

In Anspruch und Umfang stach in Deutschland das Buch des Königsberger Philosophieprofessors Ludwig Busse heraus, der hier zum bedeutendsten Vorkämpfer der Reaktion gegen den Parallelismus wurde. Busse hatte sich bereits in seinen Tokioter Vorlesungen (1887-1893) mit Erkenntnistheorie 
befasst und publizierte 1903 seine fast 500 Seiten starke Streitschrift Geist und Körper, Seele und Leib, die die Diskussion um den Parallelismus zusammenfasste und historisch platzierte: Was in der Jahrhundertmitte der Materialismusstreit gewesen war, sei heute der Parallelismusstreit (Busse 1903: 2). An diesem Streit war Busse selbst höchst beteiligt gewesen. Bereits in der Zeitschrift für Philosophie und Philosophische Kritik (Busse 1899, Paulsen 1900, König 1900, Busse 1900b) hatte er wiederholt gegen die Figur des Parallelismus und für die der Wechselwirkung argumentiert. Für Busse wie für den Großteil der Diskutanten in Deutschland stand fest, dass zwischen diesen beiden Alternativen zu entscheiden war. In seinem Buch entwickelte er seine philosophische Argumentation zunächst im Rahmen eines sich neutral gebenden Überblicks, um am Ende die Figur der Wechselwirkung als siegreich aus dem Streit hervorgehen zu lassen und seine eigene „idealistisch-spiritualistischen Weltanschauung“ (Busse 1903: 475f.) zu bekennen. Er referierte sämtliche in Umlauf befindlichen Argumente, wobei auch für ihn die Diskussion des Energieerhaltungssatzes sowie des Satzes von der Geschlossenheit der Naturkausalität von besonderer Bedeutung war, da sie die Figur des Parallelismus stützten. Busse versuchte daher nachzuweisen, dass dies keine denknotwendigen Prinzipien sind. Schon mit dem Umfang seines Werkes machte er klar, dass er alle Register zu ziehen gedachte, um die Vorzüge der Figur der Wechselwirkung gegen die des Parallelismus aufzuzeigen. Für die Wechselwirkung spreche die unabweisbare Erfahrung von Wirk- und Verursachungszusammenhängen zwischen Körper und Geist. Busse führte dafür immer wieder Beispiele an, die den Kontakt zur Empirie beschworen, deren Status allerdings höchst unausgewiesen blieb. Nicht ohne Lust an der Groteske malte er etwa die Schlacht bei Austerlitz als einen rein mechanistischen Zusammenhang von physiologischen Vorgängen aus, um die Perspektive des Parallelismus ad absurdum zu führen: Eine Darstellung, die diesem Prinzip folgen würde, dürfte nur ein blankes Getriebe von Körperbewegungen, Wahrnehmungsprozessen und Lautartikulationen skizzieren. Die psychische „Innenseite“ müsste als reine einflusslose Begleiterscheinung von der Darstellung der Verursachungsreihe ausgeschlossen bleiben. Für das „Feldherrengenie Napoleon[s]“ wäre hier kein Platz (Busse 1899: 12f.).

Ein von vielen Autoren zitiertes Beispiel war die heftige nervliche und eventuell bis zum Gehirnschlag führende Erregung, die - im Unterschied zu einem neutralen Text - das Telegramm mit der Todesnachricht einer nahestehenden Person auszulösen vermag (ebd.: 16) ${ }^{16}$. Es stützt sich auf die Offensichtlichkeit, mit der hier zwischen Wahrnehmungsakt (Lektürevorgang) und Körperreaktion ein psychisches Ereignis tritt, das als Ursache für die Erregung gelten muss. Busse hatte allerdings die größten Schwierigkeiten, die Bedeutsamkeit der zwei verschiedenen Telegrammtexte anzuerkennen: 
[D] ass der verschiedene Inhalt, der verschiedene Sinn der beiden Telegramme von irgendwelchem Einfluß auf den nervösen Vorgang sein sollte, wird man doch im Ernst nicht behaupten wollen. Gegen die Bedeutung der Schriftzeichen, die auf sie einwirken, sind die Nervenfasern ebenso gleichgiltig, wie die Telegraphendrähte gegen den Inhalt der von ihnen übermittelten Depeschen. (Busse 1899: 17f.)

Hier schiebt sich das Wernick'sche Bild über die Interpretation der Vorgänge und die Sprache beziehungsweise Schrift gerät aus dem Blick. Aber auch kein anderer der Autoren nahm die Sprache als Scharnier zwischen körperlichen und psychischen Vorgängen oder überhaupt als eigene Entität wahr, wie dies Saussure kurz darauf tat.

Auch in Frankreich wurden Busses Buch und andere Beiträge der Debatte aufmerksam rezipiert. In der von Ribot herausgegebenen Revue philosophique veröffentlichte André Godfernaux 1904 einen Aufsatz, in dem er sich mit den Konsequenzen des Parallelismus befasste. Er war von Busses Werk und dessen Kritik durch Claparède angeregt worden und offenbar noch vor Bergsons Rede geschrieben, die nicht erwähnt wird (Godfernaux 1904). 1909 ist dann auch in dieser Zeitschrift von "cette vielle querelle du parallélisme“ die Rede (Philippe 1909: 143).

In einer Festschrift für Sigwart äußerte sich neben Busse (1900a) auch der Neukantianer Heinrich Rickert zur Debatte. Er nannte die Figur, die er dem psychophysischen Parallelismus entgegenhielt, die „psychophysische Causalität" (Rickert 1900) und markierte so seine Neudeutung der alten Figur der Wechselwirkung. Die Frage der Kausalität (die ja bereits Wundt in den Titel gesetzt hatte) brach jetzt überhaupt in aller Grundsätzlichkeit auf und galt als der entscheidende Punkt, den die Figur des Parallelismus völlig ungelöst gelassen hatte. Mit Rickert nahm ein weiterer Denker, der für sein Engagement im Disziplinenstreit bekannt war, im Parallelismusstreit Stellung. Er sah in der Figur eine einseitig naturwissenschaftlich-mechanistische Bildung, gegen die er ebenso antrat wie gegen die Vorherrschaft der Naturwissenschaften überhaupt. Im Unterschied zu Stumpf verknüpfte er auf diese Weise den Parallelismusstreit eng mit dem Disziplinenstreit (der ansonsten wesentlich von dem Dilthey'schen Gegensatz zwischen Erklären und Verstehen geprägt war). Stumpf dagegen plädierte für eine Trennung der Streitpunkte:

Dieser Streit [ob Psyche und Physis kausal verknüpft sind oder eine substantielle Einheit bilden, M.W.] wird von der Definition der Natur- und der Geisteswissenschaft besser fern gehalten. Wir scheiden sie also durch die physische und die psychische Beschaffenheit ihrer Gegenstände, einerlei welches Verhältnis diese zueinander haben. (Stumpf 1907: 22)

Kennzeichnend für den neuen Stand der Figur sind die zahlreichen Dissertationen, die in diesen Jahren zum Parallelismus entstanden. Allein in Königsberg wurden mehrere Arbeiten direkt von Busse angeregt und betreut, die sich mit dem Verhältnis einzelner Autoren zum Parallelismus befassten: über Wundts Stellung zum psychophysischen Parallelismus (Mohilewer 1901), über 
Schopenhauers (Michaelis 1903) und Eduard von Hartmanns Stellung zum psychophysischen Parallelismus (Chuseau 1905). Auch an anderen philosophischen Fakultäten wurde zum Thema promoviert, wobei sowohl Arbeiten gegen den Parallelismus als auch solche für ihn verfasst wurden. So wurde etwa unter Rehmke eine kritische Arbeit (Siekermann 1899) verfasst, und die Figur wurde außerdem vom Standpunkt der Energetik her kritisiert (Spaulding 1900). In zwei Arbeiten in Basel (Reiff 1901) und Berlin (Herzberg 1913) findet sich dagegen ein positives Urteil über den Parallelismus. Die Debatte war in den Stand akademischer Schulfragen gerückt. Insgesamt hatte die Figur nun enorm an Macht verloren. Keiner konnte sich mehr ohne weiteres auf sie als Aushängeschild für die moderne wissenschaftliche Ausrichtung seines Arbeitens berufen. Doch erst der Verlust der Evidenz hatte den Weg zu der intensiven philosophischen Befragung freigemacht, die nun einsetzte und an deren Durcharbeitung eine neue Generation geschult wurde.

\section{Noch einmal: die Sprache}

Der eigenwilligste Beitrag in der damaligen Debatte stammt von dem Sprachkritiker und Autodidakten Fritz Mauthner. Er äußerte sich 1901 im ersten Band seiner Beiträge zu einer Kritik der Sprache ausführlich und recht bissig zum Parallelismus. Dabei zeigte er sich als guter Kenner der Debatte und nahm als einer der wenigen in Deutschland auch auf die britischen Beiträge Bezug (Mauthner 1923 [1901]: 290, 293). Entscheidend ist aber, dass er in seinen Ausführungen die Sprachlichkeit der Figur in den Blick nahm. Mauthner bezeichnete den psychophysischen Parallelismus freilich nicht als Signifikant (Saussures Terminus gab es noch gar nicht), die Trennung zwischen Wort und Vorstellung beachtete er ausgesprochen aufmerksam:

Fast alle unsere namhaften Philosophen, Physiologen und Psychologen bekennen sich zur Fahne des Parallelismus. Zum Worte. Ich wähle absichtlich solche Ausdrücke, weil die Weltanschauung dieser Bekenner übrigens nicht die gleiche ist. (Mauthner 1923 [1901]: 292)

Sein Vorwurf gegen den Parallelismus traf dabei mit dem Haupteinwand der meisten Kritiker zusammen: Die Figur sei eine Scheinlösung, sie blende das Problem der Ursache, das heißt die Kausalitätsfrage aus. Mauthner formulierte es nur etwas nachdrücklicher, wenn er das Aufschrecken der Parallelisten vor dem Begriff der Kausalität persifliert: „,Ursache Gott bewahre [...], ,zugeordnete، Funktion, Gott sei Dank." (Ebd.: 280) Er kommt zu dem Schluss: „Der Parallelismus umgeht den Kausalitätsbegriff، (Ebd.)

Aber Mauthner öffnete vor allem eine andere Dimension in der Debatte. $\mathrm{Zu}$ den Grundsätzen seiner Sprachkritik gehört die Annahme, dass die Sprache „wesentlich materialistisch, sensualistisch“ sei (ebd.: 235), da ihr Bildervorrat immer aus der Außenwelt stamme: Es sei in der Sprache überhaupt 
nur, was durch die Sinne gegangen ist. Da die Sinne sich aber nicht nach innen richteten, fehle für das Innenleben der Vorstellungsschatz. Hierzu gebe es - so Mauthners Grundannahme - keinen direkten Zugang. Er stand damit (wie im Übrigen auch Wilhelm Jerusalem und Freud) in scharfem Gegensatz zur allgemein anerkannten Behauptung der Unmittelbarkeit der inneren Wahrnehmung, wie sie von Dilthey und Wundt vertreten wurde. Diese unmittelbare Zugänglichkeit des Seelenlebens galt als Argument für den besonderen Status der Psychologie. Für Mauthner dagegen war klar:

Es gibt keine Psychologie, weil wir für die inneren Vorgänge keine wissenschaftliche Terminologie besitzen. Erst aus einer Kritik der Sprache könnten vielleicht einige Anfangsgründe einer künftigen Psychologie entstehen. (Ebd.: 236)

Da mit der Figur des Parallelismus eine Kongruenz zwischen Innen und Außen behauptet werde, die gar nicht existiere, hielt Mauthner sie für ungeeignet, die wirklichen Zusammenhänge zu beleuchten. „In der Freude darüber, dass in diesen Erklärungen Seele und Leib nicht mehr als Substantive oder Substanzen vorkommen", habe man sich auf ein, wie er fand, denkbar schlechtes Bild geeinigt (ebd.: 279). Dabei war Mauthners Problem nicht die Fiktionalität solcher Bilder. Um die Konstruktion komme das Denken hier gar nicht herum.

Wir besitzen aber nur eine einzige arme Sprache und quälen uns umsonst, an ihren Krücken den Abgrund zwischen Physiologie und Psychologie zu überspringen. Nur Metaphern bietet uns die Sprache, nur Bilder (ebd.: 289).

Er begrüßte daher den Standpunkt derer, für die der Parallelismus „eine bewusste Fiktion“ (ebd.: 292) sei. Aber wenn schon Fiktionen, dann sei ihm „das einfältige Märchen vom Okkasionalismus [...] immer noch lieber als das allegorische Märchen von der praestabilierten Harmonie oder als die tantenhafte Parabel vom Parallelismus" (ebd.: 284).

Keine dieser Figuren räume jedoch die Grundschwierigkeit beiseite, die Mauthner in der Natur der Sprache selbst liegen sah: Erst die Sprache schafft die Differenzen, an deren Auflösung sie dann scheitere. In „der sprachlosen Naturwirklichkeit" lassen sich, so Mauthner, die bald physiologisch, bald psychologisch betrachteten Vorgänge als „identisch“ denken, aber „in der Menschensprache“ bleiben sie doch „disparat“ (ebd.: 291). Diese Schwierigkeit, die in der sprachlichen Natur der menschlichen Erkenntnis liegt, bildete für ihn den unhintergehbaren Ausgangspunkt aller Reflexion. Von ihr ausgehend interpretierte er auch Du Bois Reymonds ,Ignorabimus' neu, indem er diese Position auf das Sprachproblem zurückführte:

Das wahre Verhältnis zwischen Seele und Leib werden wir niemals erkennen, aber nur darum nicht, weil wir uns mit unserem Denken oder mit unserer Sprache niemals auf beide Seiten zugleich stellen können. Wer die Frage nach dem Verhältnis von Seele und Leib auf- 
wirft wie andere Wissensfragen, der verkennt das Wesen der möglichen Erkenntnis. (Ebd.: 291)

Die Figur des psychophysischen Parallelismus, deren Konjunktur und Fall im ausgehenden 19. Jahrhundert in diesem Beitrag nachgezeichnet wurden, war charakterisiert durch das verwirrend große Spektrum ihrer Gebrauchsweisen. Sie war zu sehr unterschiedlichen Allianzen tauglich: Mit der Einheitsvorstellung des Monismus ließ sie sich ebenso verknüpfen wie auch mit dem Gegenteil, dem Differenzdenken des Dualismus; sie konnte mit einer materialistischen oder mit einer idealistischen Grundauffassung einhergehen, mit dem Lokalisationismus wie mit seiner Kritik; den Epiphänomenalimus stützte sie ebenso wie auch umgekehrt die Verteidigung der Eigengesetzlichkeit psychischer Vorgänge, und als eine Figur der Bescheidung war sie genauso denkbar wie als eine des Panpsychismus. Statt diese Gemengelage anzuprangern oder begriffshistorisch zu begradigen, ging es hier darum, gerade die Wendungen der Figur zu verfolgen, die eine Zeit lang an einer privilegierten und zugleich fragilen Stelle positioniert war. Diese "heiße Zone bildete ein Symptom der Umordnung des Wissens. Gerade indem sie die Differenzen und Unvereinbarkeiten in diesem Feld verdeckte, hielt sie als eine allgemein zirkulierende Figur das Feld zusammen und bündelte die Kräfte. Die breit aufgefächerte Darstellung ihres Gebrauchs war nötig, um diese, gedeckten Differenzen' zu entfalten.

Für alle Protagonisten und sogar für die meisten Kritiker war der Parallelismus dabei ein Zeichen des wissenschaftlichen Fortschritts. Er war dies jedoch weniger aufgrund einer ihm ,inhärenten' Wissenschaftlichkeit. Die Figur funktionierte vielmehr aufgrund eines Ausschlusses für den Fortschritt der Wissenschaften: Sie hat die wissenschaftliche Reflexion an dieser Stelle von der Frage der Ursache befreit. Historisch gesehen ersetzte sie die Figuren der Wechselwirkung, die in der Romantik vorherrschend gewesen waren (Welsh 2003). In systematischer Hinsicht erlaubte sie das Kausalitätsproblem am Übergang zwischen Psyche und Physis überhaupt zu suspendieren: Der Parallelismus behauptete in seinen unterschiedlichen Ausformulierungen an dieser Nahtstelle eine Einheit (Monismus) oder er ließ eine Leere, in der entweder keine Wirkungen vor sich gehen oder dem Wissen unzugängliche Umsetzungsprozesse stattfinden sollten. Als die romantische Naturphilosophie, von der man sich einst abgegrenzt hatte, weit weg in die historische Tiefe des 19. Jahrhunderts gerückt war und als allgemein überwunden galt, trat die Frage des „Wohin“ dieses Fort-Schritts deutlicher und trennender auf den Plan, und die nun einsetzende Reflexion öffnete die ungelösten Fragen erneut - daher schließlich das Brüchigwerden der Figur des Parallelismus.

In der Tat muss wohl jede Episteme das Verhältnis von Psyche und Physis erneut für sich formulieren. Es bildet eine sensible Stelle des Wissens, die 
sich zur Erhitzung eignet, und steht weder außerhalb der Geschichte noch jenseits der Diskurse.

\section{Danksagung}

Der Text ist aus meiner Arbeit am Zentrum für Literatur- und Kulturforschung Berlin im Forschungsprojekt Leonardo-Effekte, Exemplarische Konstellationen zur Trennungsgeschichte von Natur- und Geisteswissenschaften hervorgegangen, dessen Mitstreitern Caroline Welsh, Christina Brandt, Karl-Heinz Barck und Bernhard Dotzler ich für Anregungen danke. Ebenfalls möchte ich den anonymen Gutachtern von NTM für ihre anregende Kritik danken.

Open Access This article is distributed under the terms of the Creative Commons Attribution Noncommercial License which permits any noncommercial use, distribution, and reproduction in any medium, provided the original author(s) and source are credited.

\section{Anmerkungen}

1 Im Archiv für Begriffsgeschichte verfolgt Helmut Hildebrand den Parallelismus bis in die Gegenwart. Die Figur bleibt bei ihm aber allgemeiner gefasst und ist nicht an den Wortlaut gebunden, auch setzt er ihr Auftauchen später an, da er sie erst ab Wundts Aufsatz zum Parallelismus 1894 zur Kenntnis nimmt (Hildebrand 1985, 1989). Auch Michael Heidelberger kann Vertreter des psychophysischen Parallelismus nach 1900 benennen, die Figur wird bei ihm von der Fechner'schen Fassung aus verallgemeinert (Heidelberger 2000).

2 Ich entlehne diesen Begriff von Michel Foucault (1989 [1984]: 13). Jede Episteme hat ihre eigenen Wahrheitsspiele. Der Anklang an Wittgensteins Terminus des "Sprachspiels“ ist beabsichtigt.

3 Die Diskussion um die Beschaffenheit der Elektrizität - ob sie materielle oder immaterielle Kraft sei - eröffnete allerdings zur gleichen Zeit ein Feld, dessen Übereinstimmung mit dieser Grundannahme noch keineswegs abgemacht war.

$4 \mathrm{Zu}$ der rege geführten Diskussion um Metaphern im wissenschaftlichen Kontext s. Brandt 2004: $28 f$.

5 An dem romantischen Anspruch, eine umfassende Theorie von der „Einheit der Welt, der Realität wie der Ideenwelt" (Arendt 1999: 29) auszuarbeiten, hat Fechner bei allem Wandel lebenslang festgehalten.

6 Diesen von ihm als rein wissenschaftlich bezeichneten Standpunkten stellt er im ZendAvesta drei außerwissenschaftliche zur Seite, die er in der Psychophysik dann nicht mehr anführt. Es handelt sich um den „wechselnden (natürlichen)“, den „nicht unterscheidenden (ursprünglich naturwüchsigen)“ und den „mischenden (gemeinen)“ Standpunkt (Fechner 1851, Bd. 2: 349f.).

7 Bei Spencer heißt es dort: „I am merely showing a parallelism between a certain evolution and the correlative psychical evolution." (Herv. i. O.) Vgl. Jackson: „Mental states are only concomitant with nervous states." (1971a [1866]: 355)

8 Leider präzisiert Vidoni hier seine Quelle nicht.

9 Aufgrund der ungenauen Übersetzung hier das frz. Original: „Supposez un livre écrit dans une langue originelle et muni d'une traduction interlinéaire; le livre est la nature, la langue originale est l'évènement morale, la traduction interlinéaire est l'évènement physique, et 
l'ordre des chapitres est l'ordre des êtres." (Taine 1878 [1870]: 334). ,Une langue originelle‘ oder ,originale ‘ wäre wörtlich eine ,ursprüngliche Sprache‘ oder ,Ursprache‘, l'ordre‘ wäre ,Ordnung‘. Die Übersetzung von ,morale‘ mit ,psychisch` war allgemein üblich.

10 Auch bei Freud ist die Sprache also ein Apparat, aber nicht allein ein Übertragungsmedium wie der Wernick'sche Telegraph, sondern auch ein Speichermedium, welches zudem das, was es überträgt und speichert, überhaupt erst konstituiert: sprachliche Realität.

11 Der Cours de linguistique générale wurde 1916 von Schülern Saussures herausgegeben, die den Text edierten. Wie die kritischen Ausgaben von Engler u.a. zeigten, haben die Herausgeber den Text an zahlreichen Stellen ergänzt und verändert. Auch diese Passage ist ein Zusatz der Herausgeber (Saussure 1995: 467). Es bleibt also offen, ob Saussure selbst einmal die Formulierung des Parallelismus explizit gebrauchte, die sein Vortrag hier immerhin evoziert hat. Für die Wirkungsgeschichte ist die 1916 publizierte, hier zitierte Textfassung aber entscheidend.

12 Als Wundt 1879 in Leipzig das weltweit erste psychologische Institut (Laboratorium) gründete, war er Inhaber eines philosophischen Lehrstuhls an der Universität.

13 Anwesend waren u.a. Alfred Binet, Wladimir Bechterew, Hermann Ebbinghaus, Sigmund Exner, Paul Flechsig, Auguste Forel, Ewald Hering, Pierre Janet, Hugo Münsterberg, Charles Richet, Henry Sidgwick. Der Mitbegründer Théodule Ribot und die Mitglieder Wilhelm Wundt und William James waren auf diesem Kongress nicht zugegen (Anonym 1897: VIIXLI), während zum Beispiel Sigmund Freud als Nichtmitglied teilnahm (Freud 1987: 178).

14 Im Text steht hier ausgerechnet fälschlich „das Physische“. Diese Vertauschung findet sich auch in der 2. überarbeiteten Publikation der Rede (Stumpf 1903: 25).

$15 \mathrm{Zu}$ den Ungleichzeitigkeiten der Debatte gehört, dass Fechner einen solchen Gedanken auf seine Weise - schon früh formuliert hatte, ohne dass er in der Folge Gewicht bekam: „Die lebendige Kraft, die zum Holzhacken verwandt wird, und die lebendige Kraft, die zum Denken, das ist zu den unterliegenden psychophysischen Processen verwandt wird, sind nach Vorigem quantitativ nicht nur vergleichbar, sondern selbst in einander umsetzbar, und hiermit beide Leistungen selbst nach körperlicher Seite durch einen gemeinsamen Massstab messbar." (Fechner 1860, Bd. 1: 43)

16 Busse verweist an dieser Stelle darauf, dass das Beispiel bereits von Friedrich Albert Lange in seiner Geschichte des Materialismus und von Franz Erhardt 1897 in Die Wechselwirkung zwischen Leib und Seele angeführt wurde. Auch Heinrich Rickert nimmt es auf, spricht ihm aber eine geringere Überzeugungskraft als Busse zu (Rickert 1900: 68).

\section{Literatur}

Anonym, 1895: Parallelismus, pschophysischer. In: Brockhaus. Konversations-Lexikon (14. völlig neubearb. Aufl.). Bd. 12. Leipzig u.a.: Brockhaus, S. 887.

Anonym, 1897. Acten des dritten internationalen Congresses für Psychologie in München 1896. München: Lehmann.

Arendt, Hans-Jürgen, 1999. Gustav Theodor Fechner, ein deutscher Naturwissenschaftler und Philosoph im 19. Jahrhundert. Frankfurt a. M.: Peter Lang Verlag.

Ash, Mitchell G., 1999. Psychologie in Deutschland um 1900. Reflexiver Diskurs des Bildungsbürgertums, Teilgebiet der Philosophie, akademische Disziplin. In: Christoph König und Eberhard Lämmert, Hg., Kultur, Wissen und Universität um 1900. Frankfurt a. M.: Fischer.

Ash, Mitchell G., 2007. Psychological Thought and Practice. Historical and Interdisciplinary Perspectives. In: Mitchell Ash und Thomas Sturm, Hg., Psychology's Territories. Historical and Contemporary Perspectives from Different Disciplines. Mahwah, NJ/London: Lawrence Erlbaum Associates, 1-27.

Bain, Alexander, 1873 [1872]. Mind and Body. The Theories of Their Relation. Cornhill/London: Henrys King \& Co. [Nachdruck 1973].

Bain, Alexander, 1874 [1872]. Geist und Körper. Theorien über ihre gegenseitigen Beziehungen. Leipzig: Brockhaus [autorisierte Ausgabe]. 
Bain, Alexander, 1904. Autobiographie. London u.a.: Longmans Green and Co.

Bergson, Henri, 1928 [1904]. Hirn und Denken. Eine philosophische Illusion. In: Ders., Die seelische Energie. Jena: Diederichs, 171-188.

Bergson, Henri 1970 [1904]. Le cerveau et la pensée. Une illusion philosophique (Le paralogisme psychophysiologique). In: Ders., Oevres. Paris: PUF, 959-974.

Blumenberg, Hans, 1998. Paradigmen zu einer Metaphorologie. Frankfurt a. M.: Suhrkamp.

Bölsche, Wilhelm, 1976 [1886]. Naturwissenschaftliche Grundlagen der Poesie. Tübingen: Max Niemeyer.

Brandt, Christina, 2004. Metapher und Experiment. Von der Virusforschung zum genetischen Code. Göttingen: Wallstein.

Breidbach, Olaf, 1997. Die Materialisierung des Ichs. Zur Geschichte der Hirnforschung im 19. und 20. Jahrhundert. Frankfurt a. M.: Suhrkamp.

Broca, Paul, 1861. Remarques sur le Siège de la faculté du langage articulé, suivi d'une observation d'Aphémie (perte de parole). Bulletin de la société anatomique de Paris, 6, 330-357.

Brunschvicg, Léon, 1931. Physique indéterministe et parallélisme psycho-physiologique. Revue de Synthèse, 2, 31-34.

Busse, Ludwig, 1899. Leib und Seele. Zeitschrift für Philosophie und philosophische Kritik, 114, $1-26$.

Busse, Ludwig, 1900a. Die Wechselwirkung zwischen Leib und Seele und das Gesetz von der Erhaltung der Energie. In: Philosophische Abhandlungen. Christoph Sigwart zu seinem Sechzigsten Geburtstage gewidmet. Tübingen u. a.: Mohr, 89-125.

Busse, Ludwig, 1900b. Wechselwirkung oder Parallelismus? Zeitschrift für Philosophie und philosophische Kritik, 116, 56-80.

Busse, Ludwig, 1903. Geist und Körper, Seele und Leib. Leipzig: Dürr'sche Buchhandlung.

Canguilhem, Georges, 1989 [1980]. Gehirn und Denken. In: Ders., Grenzen medizinischer Rationalität: historsich-epistemologische Untersuchungen. Übers. v. Monika Noll. Tübingen: Edition Discord, 7-40.

Chuseau, Hans, 1905. Eduard von Hartmanns Stellung zum psychophysischen Parallelismus. Königsberg: O. Kümmel.

Dilthey, Wilhelm, 1974 [1894]. Ideen über eine beschreibende und zergliedernde Psychologie. In: Ders., Gesammelte Schriften, Bd. 5. Stuttgart: Teuber.

Du Bois Reymond, Emil, 1886. Die Grenzen des Naturerkennens. (Vortrag 1872). In: Ders., Reden, Bd. 1. Leipzig: Veit, 105-140.

Dupéron, Isabelle, 2000. G. T. Fechner. Le parallélisme psychophysiologique. Paris: PUF.

Elger, Christian Erich u.a., 2004. Das Manifest. Hirnforschung im 21. Jahrhundert. Gehirn und Geist, 6.

Eling, Paul, Hg., 1994. Reader in the History of Aphasia. Amsterdam u.a.: John Benjamins Publishing Company.

Fechner, Gustav Theodor, 1823. Praemissae ad theoriam organismi generalem. Leipzig: Staritz.

Fechner, Gustav Theodor, 1848. Nanna oder über das Seelenleben der Pflanzen. Leipzig: Voß.

Fechner, Gustav Theodor, 1851. Zend-Avesta. Oder über die Dinge des Himmels und des Jenseits. Vom Standpunkt der Naturbetrachtung. 3 Bde. Leipzig: Voß.

Fechner, Gustav Theodor, 1860. Elemente der Psychophysik. 2 Bde. Leipzig: Breitkopf \& Härtel.

Fechner, Gustav Theodor, 1879. Die Tagesansicht gegenüber der Nachtansicht. Leipzig: Breitkopf \& Härtel.

Foucault, Michel, 1974 [1966]. Die Ordnung der Dinge. Frankfurt a. M.: Suhrkamp.

Foucault, Michel, 1989 [1984]. Sexualität und Wahrheit. Bd. 2: Der Gebrauch der Lüste. Frankfurt a. M.: Suhrkamp.

Freud, Sigmund, 1982 [1890]. Seelenbehandlung. In: Ders., Studienausgabe. Ergänzungsband. Frankfurt a. M.: Fischer, 14-35.

Freud, Sigmund, 1987. Briefe an Wilhelm Fließ 1887-1904. Hg. v. Jeffrey Moussaieff Masson unter Mitarb. v. M. Schröter. Frankfurt a. M.: Fischer.

Freud, Sigmund, 1992 [1891]. Zur Auffassung der Aphasien. Eine kritischen Studie. Hg. v. Paul Vogel. Frankfurt a. M.: Fischer.

Freud, Sigmund/Groddeck, Georg, 1988. Briefe über das Es. Hg. v. Margaretha Honneger. Frankfurt a. M.: Fischer. 
Freud, Sigmund, 1999 [1915]. Das Unbewusste. In: Ders., Gesammelte Werke, Bd. 10. Frankfurt a. M.: Fischer, 263-303.

Gethmann, Carl Friedrich u.a., 2005. Manifest Geisteswissenschaften. Hg. v. Präsidenten der Berlin-Brandenburgischen Akademie der Wissesnschaften. Berlin.

Godernaux, André, 1904. Le parallélisme psychophysique et ses conséquences. Revue philosophique de la France et de l'etranger. 58, 329-352, 482-504. [Reprint Liechtenstein 1967].

Hagner, Michael, 1997. Homo cerebralis. Der Wandel vom Seelenorgan zum Gehirn. Berlin: Berlin Verlag.

Heidelberger, Michael, 1993. Die innere Seite der Natur. Gustav Theodor Fechners wissenschaftlich-philosophische Weltauffassung. Frankfurt a. M.: Klostermann.

Heidelberger, Michael, 2000. Fechner und Mach zum Leib-Seele-Problem. In: Andreas Arndt und Walter Jaeschke, Hg., Materialismus und Spiritualismus. Philosophie und Wissenschaften nach 1848. Hamburg: Meiner, 53-67.

Hering, Ewald, 1875. Zur Lehre von der Beziehung von Leib und Seele. Erste Mitteilung. Über Fechner's psychophysisches Gesetz. Sitzungsberichte der kaiserlichen Akademie der Wissenschaften, mathematisch-naturwissenschaftliche Classe, 71. Wien: Carl Gerold's Sohn, 310-348.

Hering, Ewald, 1878. Zur Lehre vom Lichtsinne. Sechs Mittheilungen an die Kaiserliche Akademie der Wissenschaften in Wien. Wien: Carl Gerold's Sohn.

Herzberg, Alexander, 1913. Über den Unterschied zwischen Physischem und Psychischem und über den Sinn der Wechselwirkungslehre und des Parallelismus. Berlin: Pakuscher.

Hildebrand, Helmut, 1985. Psychophysischer Parallelismus, Psychophysisches Problem und die Gegenstandsbestimmung der Psychologie. Archiv für Begriffsgeschichte, 29, 147-181.

Hildebrand, Helmut, 1989. Parallelismus, psychophysischer. In: Joachim Ritter u.a., Hg., Historisches Wörterbuch der Philosophie, Bd. 7. Basel: Schwabe, 100-107.

Jackson, John Hughlings, 1927 [1884]. Die Croon-Vorlesungen über Aufbau und Abbau des Nervensystems/Croonian Lectures on the Evolution and Dissolution of the Nerveous System. Berlin: S. Karger [zweisprachige Ausgabe].

Jackson, John Hughlings, 1971a [1866]. Notes on the Physiology and Pathology of Language. In: Ders., Selected Writings, Bd. 2, hg. v. James Taylor u. a. New York: Basic Books, 121-128.

Jackson, John Hughlings, 1971b [1874]. On the Nature of the Duality of the Brain. In: Ders., Selected Writings, Bd. 2, hg. v. James Taylor u. a. New York: Basic Books, 129-145.

Jackson, John Hughlings, 1971c [1878]. On Affections of Speech from Disease of the Brain. In: Ders., Selected Writings, Bd. 2., hg. v. James Taylor u. a. New York: Basic Books, 155-170.

Jäger, Ludwig, 2001. Neurosemiologie. Das transdisziplinäre Fundament der saussureschen Sprachidee. Cahiers de Ferdinand de Saussure. Revue suisse de linguistique générale, 54, 289-337.

Kay, Lily, 2000. Who Wrote the Book of Live? A History of the Genetic Code. Stanford: Stanford University Press.

König, Edmund, 1899 Die Lehre vom Psychophysischen Parallelismus und ihre Gegner. Zeitschrift für Philosophie und philosophische Kritik, 115, 161-192.

Latour, Bruno, 1997. Aufstand der Dinge. Die Tageszeitung, 28. Oktober 1997.

Lenoir, Timothy, 1997. Instituting Science. The Cultural Production of Scientific Disciplines. Stanford: Stanford University Press.

Leuschner, Wolfgang, 1992. Einleitung. In: Sigmund Freud, Zur Auffassung der Aphasie. Frankfurt a. M.: Fischer, 7-31.

Linke, Detlef Bernhard/Kurthen, Martin, 1988. Parallelität von Gehirn und Seele. Neurowissenschaften und Leib-Seele Problem. Stuttgart: Enke.

Mach, Ernst, 1863. Vorträge über Psychophysik. Österreichische Zeitschrift für Praktische Heilkunde, 9, 146-148, 167-170, 202-204, 225-228, 242-245, 260-261, 277-279, 294-298, 316-318, 335-338, 352-354, 362-366.

Mach, Ernst, 1906 [1886]. Analyse der Empfindungen und das Verhältnis des Physischen zum Psychischen. Jena: Gustav Fischer.

Mauthner, Fritz, 1923 [1901]. Beiträge zu einer Kritik der Sprache. Bd. 1: Zur Sprache und zur Psychologie. Leipzig: Felix Meiner [3. Aufl., um Zusätze vermehrt]. 
Michaelis, Heinrich, 1903. Schopenhauers Stellung zum psychophysischen Parallelismus. Königsberg: Hartung.

Mill, John Stuart, 1877 [1843]. System der deduktiven und induktiven Logik. Zweiter Teil, übers. v. J. Schiel. Braunschweig: Friedrich Vieweg und Sohn.

Misler, Nicoletta, 1997. A Citadel of Idealism. RAKhN as a Sovjet Anomaly. In: John E. Bolt u.a., Hg., Experiment. A Journal of Russian Culture, Bd. 3. Los Angeles: Charles Schlacks, Jr. Publisher.

Mohilewer, Joseph, 1901. Wundts Stellung zum psychophysischen Parallelismus. Königsberg: H. Jäger.

Paulsen, Friedrich, 1900. Noch ein Wort zur Theorie des Parallelismus. Zeitschrift für Philosophie und philosophische Kritik, 115, 1-8.

Philippe, Jean, 1909. Pour et contre la Psychophysique. Revue philosophique de la France et de l'etranger, 68, 113-149 [Reprint Liechtenstein 1967].

Pohlenz, Gerd, 1977. Das parallelistische Fehlverständnis des Physischen und des Psychischen. Meisenheim: Hain.

Radkau, Joachim, 1998. Das Zeitalter der Nervosität. München/Wien: Hanser.

Reiff, Paul, 1901. Der moderne psychophysische Parallelismus. Heilbronn: Schell.

Rickert, Heinrich, 1900. Psychophysische Causalität und psychophysischer Parallelismus. In: Philosophische Abhandlungen. Christoph Sigwart zu seinem Sechzigsten Geburtstage gewidmet. Tübingen u.a.: Mohr, 59-87.

Saussure, Ferdinand de, 1967 [1916]. Grundfragen der allgemeinen Sprachwissenschaft. Hg. v. Charles Bally, Albert Sechehaye, übers. v. Herman Lommel. Berlin: de Gruyter.

Saussure, Ferdinand de, 1995 [1916]. Cours de linguistique générale. Hg. v. Charles Bally, Albert Sechehaye. Kritische Edition v. Tullio de Mauro. Paris: Payot \& Rivages.

Scheerer, Eckart, 1993. Gustav Theodor Fechner und die Neurobiologie: „Innnere Psychophysik“ und „tierische Elektrizität“. In: Ernst Florey und Olaf Breidbach, Hg., Das Gehirn - Organ der Seele? Zur Ideengeschichte der Neurobiologie. Berlin: Akademie-Verlag, 259-288.

Schmidt, Nicole D., 1995. Philosophie und Psychologie. Trennungsgeschichte, Dogmen, Perspektiven. Reinbek: Rowolth.

Senft, Harald, 1978. J.H. Jacksons Beitrag zur Neuropsychologie. Bonn: o. V.

Siegert, Bernhardt, 1999. Das Leben zählt nicht. Naturwissenschaft und Geisteswissenschaft bei Dilthey aus mediengeschichtlicher Sicht. In: Claus Pias, Hg., Medien. Dreizehn Vorträge zur Medienkultur. Weimar: Verl. u. Datenbank für Geisteswissenschaften, 161-182.

Siekermann, Franz, 1899. Zur Kritik der Hypothese des psychophysischen Parallelismus. Greifswald: Kunike.

Spaulding, Eduard Gleason, 1900. Beiträge zur Kritik des psychophysischen Parallelismus vom Standpunkte der Energetik. Bonn: Hochschulschriften.

Spencer, Herbert, 1885 [1855]. The Principles of Psychology. 2 Bd. New York: D. Appelton and company. 4., stark umgearbeitete Aufl. [Reprint BookSurge Publishing 2000].

Steiner, Andreas, 1964. Das nervöse Zeitalter. Zürich: Juris.

Stumpf, Carl, 1897. Eröffnungsrede zum 3. Internationalen Kongress für Psychologie in München 1896. Acten des dritten internationalen Congresses für Psychologie in München 1896. München: Lehmann, 3-16.

Stumpf, Carl, 1903. Leib und Seele. In: Ders.: Zwei Reden. Leipzig: J. A. Barth, 5-38.

Stumpf, Carl, 1907. Zur Einteilung der Wissenschaften. Berlin: Akademieverlag.

Stumpf, Carl, 1919. Spinozastudien. Abhandlungen der preussischen Akademie der Wissenschaften, 4, 1-57.

Taine, Hippolyte, 1878 [1870]. De L'intelligence. 2 Bde. Paris: Hachette.

Taine, Hippolyte, 1880 [1870]. Der Verstand. 2 Bde. Bonn: Strauß.

Tesak, Jürgen, 2001. Geschichte der Aphasie. Idelstein: Schulz-Kirchner.

Tyndall, John, 1871 [1868]. Scientific Materialism. In: Ders., Fragments of Science for Unscientific People. London: Longmans, Green and Co., 109-123.

Vidoni, Ferdinando, 1991. Ignorabimus, Emile du Bois-Reymond und die Debatte über die Grenzen wissenschaftlicher Erkenntnis im 19. Jahrhundert. Frankfurt a. M. u.a.: Peter Lang.

Wegener, Mai, 2004. Neuronen und Neurosen. Der psychische Apparat bei Freud und Lacan. Ein historisch-theoretischer Versuch zu Freuds Entwurf von 1895. München: Fink. 
Wegener, Mai, 2005. Das psychophysische Unbewußte. Gustav Theodor Fechner und der Mond. In: Michael B. Buchholz und Günter Gödde, Hg., Macht und Dynamik des Unbewußten. Auseinandersetzungen in Medizin Philosophie und Psychoanalyse, Bd. 1. Gießen: Psychosozial Verlag, 247-267.

Wegener, Mai, 2006. Heiße Zonen. Psychophysischer Parallelismus. In: Sigrid Weigel, Hg., Trajekte Extra: 10 Jahre Zentrum für Literatur und Kulturforschung. Berlin: Zentrum für Literatur- und Kulturforschung, 65-67.

Wegener, Mai, 2008. „...das Gefühl Liebe entspräche einer rechtsdrehenden Spiralbewegung der Hirnmoleküle..." Zur Figur des psychophysischen Parallelismus im ausgehenden 19. Jahrhundert. In: Caroline Welsh und Stefan Willer, Hg., "Interesse für bedingtes Wissen“. Wechselbeziehungen zwischen den Wissenskulturen. München: Fink, 131-151.

Welsh, Caroline, 2003. Hirnhölenpoetiken. Theorien zu Wahrnehmung in Wissenschaft, Ästhetik und Literatur um 1800. Freiburg i.B.: Rombach.

Wernicke, Carl, 1893 [1874]. Der Aphasische Symptomkomplex. In: Ders., Gesammelte Aufsätze und kritische Referate zur Pathologie des Nervensystems. Berlin: Fischer's Medicinische Buchhandlung, 1-70.

Worbs, Michael, 1983. Nervenkunst. Frankfurt a. M.: Europäische Verlagsanstalt.

Wundt, Wilhelm, 1894. Ueber psychische Causalität und das Princip des psychophysischen Parallelismus. Philosophische Studien, 10, 1-124.

Ziehen, Theodor, 1911 [1890]. Leitfaden der physiologischen Psychologie. Jena: G. Fischer [9., teilw. umgearbeitete Aufl.].

Ziehen, Theodor, 1923. Theodor Ziehen. In: Raymund Schmidt, Hg., Die Philosophie der Gegenwart in Selbstdarstellungen, Bd. 4. Leipzig: Felix Meiner, 218-236.

\author{
Mai Wegener \\ Max-Planck Institut für Wissenschaftsgeschichte \\ Boltzmannstr. 22 \\ D-14195 Berlin \\ E-Mail: mai.wegener@pasberlin.de
}

